\title{
An analysis of key characteristics of the Frank-Read source process in FCC metals
}

\author{
Shuozhi Xu ${ }^{\mathrm{a}, *}$, Liming Xiong ${ }^{\mathrm{b}}$, Youping Chen $^{\mathrm{c}}$, David L. McDowell ${ }^{\mathrm{a}, \mathrm{d}}$ \\ ${ }^{a} G W W$ School of Mechanical Engineering, Georgia Institute of Technology, Atlanta, GA \\ 30332-0405, USA \\ ${ }^{b}$ Department of Aerospace Engineering, Iowa State University, Ames, IA 50011, USA \\ ${ }^{c}$ Department of Mechanical and Aerospace Engineering, University of Florida, Gainesville, \\ FL 32611-6250, USA \\ ${ }^{d}$ School of Materials Science and Engineering, Georgia Institute of Technology, Atlanta, GA \\ 30332-0245, USA
}

\begin{abstract}
A well-known intragranular dislocation source, the Frank-Read (FR) source, plays an important role in size-dependent dislocation multiplication in crystalline materials. Despite a number of studies in this topic, a systematic investigation of multiple aspects of the FR source in different materials is lacking. In this paper, we employ large scale quasistatic concurrent atomistic-continuum (CAC) simulations to model an edge dislocation bowing out from an FR source in $\mathrm{Cu}, \mathrm{Ni}$, and $\mathrm{Al}$. First, a number of quantities that are important for the FR source process are quantified in the coarse-grained domain. Then, two key characteristics of the FR source, including the critical shear stress and critical dislocation configuration, are investigated. In all crystalline materials, the critical stresses and the aspect ratio of the dislocation half-loop height to the FR source length scale well with respect to the FR source length. In Al, the critical stress calculated by CAC simulations for a given FR source length agrees reasonably well with a continuum model that explicitly includes the dislocation core energy. Nevertheless, the predictions of the isotropic elastic theory do not accurately capture the $\mathrm{FR}$ source responses in $\mathrm{Cu}$ and $\mathrm{Ni}$, which have a relatively large stacking fault width and elastic anisotropy. Our results highlight
\end{abstract}

\footnotetext{
${ }^{*}$ Corresponding author
} 
the significance of directly simulating the FR source activities using fully 3D models and shed light on developing more accurate continuum models.

Keywords: Concurrent atomistic-continuum, dislocation multiplication, Frank-Read source, FCC metals

\section{Introduction}

Plastic deformation increases the dislocation content of a crystal, a process termed dislocation multiplication (Weertman and Weertman, 1992). Among many possible mechanisms such as Bardeen-Herring climb source and multiple cross glide mechanism, the Frank-Read (FR) source is usually considered the most important in generating dislocations in the bulk of a crystalline material (Hirth and Lothe, 1992). In a polycrystalline face-centered cubic (FCC) metal, an FR source can produce a series of dislocations on one slip plane, which then may pile up against a grain boundary (GB) and manifest grain size-dependent metal plasticity (Ramesh, 2009). As more dislocations are generated from the FR sources, size-scaling hardening is exhibited due to an increasing back stress (Szajewski et al., 2013). In stage I work hardening, the scarcity of interfering dislocations allows operation of multiple FR sources, which is responsible for a majority of the rise in dislocation density, especially at low temperatures in the absence of dislocation climb (Kuhlmann-Wilsdorf, 2002). Compared with other dislocation sources such as homogeneous nucleation or dislocation nucleation from GBs and precipitates, FR sources have a larger characteristic length and a lower critical shear stress to activate (Shimokawa and Kitada, 2014). At strain rates greater than $10^{8} \mathrm{~s}^{-1}$, however, the strength of $\mathrm{FR}$ sources no longer controls the yield point of a crystalline metal because they are unable to operate before homogeneous dislocation nucleation relaxes elastic stresses (GurrutxagaLerma et al., 2015). FR sources are suppressed in stage II work hardening because dislocations on multiple slip systems interact with each other and reduce the mean free path of dislocations.

Prior to the discovery of the FR source (Frank and Read, 1950), the prob- 
lem of a dislocation bypass of an array of obstacles was studied using line tension (Mott and Nabarro, 1948). The line tension for paired obstacle bypass, introduced by Orowan (Martin, 1998) to balance the glide force on a curved dislocation, is considered a parameter of the configurational force on an entire dislocation line segment (Hirth and Lothe, 1992), and is given by

$$
\tau_{\mathrm{OR}}=\frac{\mu b}{L}
$$

where $\mu$ is the isotropic shear modulus, $b$ is the Burgers vector of a full dislocation, and $L$ is the distance between two non-shearable obstacles. Taking into account the elastic interactions between different segments (Bacon, 1967), the bowing of a dislocation segment was studied directly using self-stress by Brown (1964). Foreman (1967) discretized curved dislocations into short straight segments and considered the self-stress at each node; the critical stress for dislocation bow-out from a pointlike FR source is expressed as

$$
\tau_{\mathrm{FO}}=\frac{A \mu b}{2 \pi L}\left[\ln \left(\frac{L}{r_{0}}\right)+B\right]
$$

where $A$ and $B$ are parameters, $r_{0}$ is the full dislocation core radius. Elastic theory predicts that $A=1$ and $A=1 /(1-\nu)$ for edge and screw dislocations, respectively, where $\nu$ is the isotropic Poisson's ratio; $B$, which is difficult to estimate in the line tension model, is associated with the dislocation core field. We remark that for curved or finite dislocation segments, $r_{0}$ sometimes refers to the cut-off radius for dislocation interaction. In this paper, we assume this cutoff radius equals the core radius as they both correlate positively with dislocation core energy.

Bacon et al. (1973) and Scattergood and Bacon (1982) addressed another problem of a dislocation bypassing a periodic array of finite size obstacles and calculated its critical stress, i.e.,

$$
\tau_{\mathrm{SB}}=\frac{A \mu b}{2 \pi L}\left[\ln \left(\frac{\bar{D}}{r_{0}}\right)+B\right]
$$

where $L$ is the length of the initial dislocation segment, $\bar{D}$ is the harmonic mean of $L$ and the obstacle diameter $D$, i.e., $\bar{D}=\left(D^{-1}+L^{-1}\right)^{-1}$. Let the obstacles 
be precipitates or voids, $B=0.7$ and 1.52 , respectively. Compared with previous models with pointlike obstacles, free surfaces at voids are accounted for in Eq. 3; for precipitates, dislocations are not allowed to move around the obstacle periphery.

All these models assume that dislocations move at an infinitesimal speed (Ghoniem et al., 2000). At strain rates beyond $10^{6} \mathrm{~s}^{-1}$, the static continuum theories need to be modified to include inertia effects on the motion of dislocations whose speed exceeds the domain of validity of the linear viscous drag law (Bitzek and Gumbsch, 2004). Another major assumption is that dislocations bypass of obstacles following the Orowan bypass mechanism (Friedel, 1964), i.e., a dislocation bows out greatly to leave a loop on the same slip plane around the impenetrable obstacle. As a result, these models are applicable for the process of a dislocation bowing-out from an FR source. Models based on other mechanisms, e.g., Hirsch bypass mechanism (Hatano, 2006) and shearing mechanism (Hull and Bacon, 2011), however, do not fit the FR source process and may yield incorrect critical stresses. As will be shown later, these two assumptions stated above are satisfied in our simulations to make our results comparable to the continuum models.

Accurately accounting for the image forces on the dislocation due to the void's free surface, Crone et al. (2015) refined Eq. 3 and proposed a new model of obstacle strengthening based on dislocation dynamics (DD), i.e.,

$$
\tau_{\mathrm{CMK}}=\frac{A \mu b}{2 \pi(L+D / 2)} \ln \left(\frac{\bar{D}}{r_{0}}\right) .
$$

We remark that special treatments are necessary in DD to remove the singularities of stress fields and elastic energy at dislocation core center (Cai et al., 2006). Although the DD method can be informed atomistically (Martínez et al., 2008; Lehtinen et al., 2016), a dislocation in a crystal is intrinsically discrete and atomistic; its dynamics is governed by a classical physics which may not be reducible to continuum theory (Cottrell, 2002). Szajewski et al. (2015) found that even after fitting parameters to match the atomistic line tension, the standard DD technique still can't match the atomistic configurations for the screw 
FR source. Thus, atomistic simulations are desirable in capturing the nonlinear dislocation core field (Lothe and Hirth, 2005), compared to continuum approaches such as DD, the phase field method (PFM) (Berry et al., 2014), and level-set methods (Xiang et al., 2003). In the last decade, a number of MD simulations were performed to explore a dislocation bowing-out from an FR source (de Koning et al., 2003; Li and Yang, 2006; Shimokawa and Kitada, 2014). In these calculations, periodic arrays of dislocation (PAD) models are usually employed to impose an infinite array of obstacles and an infinitely long dislocation, where the simulation supercell has a limited size (Bacon et al., 2009). Accompanied by non-negligible spurious image forces up to half of the true applied force, the PAD models overestimate the critical shear stress of an FR source (Szajewski and Curtin, 2015). Thus, an ideal simulation of the FR source process needs to accurately capture both the core effect at the nano-scale and the long range stress fields of the dislocation loop at the submicron-scale, using a sufficiently large model free of image forces. Recently, an attempt has been made to explore the dislocation bow-out configuration via an image-free multiscale simulation using the coupled atomistic/discrete dislocation (CADD) method (Szajewski et al., 2015). Nevertheless, a systematic study of multiple aspects of the FR sources in different metals is still lacking, to the best of our knowledge.

In this paper, we perform concurrent atomistic-continuum (CAC) simulations in the quasistatic, athermal limit to investigate the process of an edge dislocation bowing-out from an FR source. Employing a unified atomistic-continuum integral formulation with discontinuous elements, CAC admits propagation of dislocations and intrinsic stacking faults through a lattice in both atomistic and coarse-grained domains with the underlying interatomic potential as the only constitutive relation (Chen, 2009). Within each element, a finite element method with Gaussian quadrature is used to calculate the force/energy of the integration points and update the nodal positions, from which the positions of atoms inside the element are interpolated (Xiong et al., 2011, 2016). The non-singular dislocation core structure/energy and Burgers vector are naturally 
accommodated in CAC to yield an accurate generalized stacking fault energy (GSFE) surface (Xu et al., 2015). Previously, CAC was used to model nucleation and growth of dislocation loops in $\mathrm{Cu}, \mathrm{Al}$, and $\mathrm{Si}$ (Xiong et al., 2012b), dislocation-void interactions in Ni (Xiong et al., 2015), dislocation-GB interaction in $\mathrm{Cu}$ and $\mathrm{Al}$ (Xu et al., 2016b), and dislocations bowing out from obstacles in $\mathrm{Al}$ (Xu et al., 2016a). The success of these calculations, in which adaptive mesh refinement is unnecessary (Xu et al., 2016c), suggests the viability of using CAC simulations to study FR source operation in a sufficiently large model with negligible image effects. We will focus on the critical shear stress and critical dislocation configuration - two key characteristics of the FR source process that can be used as a set of constitutive rules in DD simulations (Benzerga, 2009). Three FCC metals, $\mathrm{Cu}, \mathrm{Ni}$, and $\mathrm{Al}$ will be studied because of their different $\mathrm{SFE}$ and elastic anisotropy index (Ramesh, 2009). From the methodological viewpoint, we will show that $\mathrm{CAC}$ is able to simulate 3D dislocation loop generation

from an FR source, a benchmark problem for multiscale simulations and one of the building blocks for more complicated 3D dislocation substructure evolution (Cantrell, 2004). Moreover, the fact that the FR source process involves both elastic interactions between dislocations as well as atomistic details makes it a good "test problem" to validate the CAC method by comparing our results with those predicted by continuum models.

\section{Methodology}

The operation of an FR source with voids as pinning obstacles is a complex process. Initially, a straight dislocation line is pinned between two voids; then subject to a shear stress, the segment begins to bow out until the critical configuration is reached, as shown in Fig. 1(a-c). Note that both ends of the dislocation move around the void periphery. If the applied shear stress is larger than the critical shear stress, the bowed-out dislocation will continue to grow until two parts of the "kidney shaped" segments collide to form a dislocation loop, leaving behind a new, straight dislocation line pinned between two voids, 
as shown in Fig. 1(d-e). A successful modeling technique for this problem needs to reasonably accurately predict (1) stress fields of dislocations, (2) dislocation dissociation, (3) Peierls stress, (4) dislocation core energy, (5) image forces on dislocations at a void surface, (6) stress concentration around the void, (7) surface energy of a void, and (8) elastic anisotropy of crystals. For CAC, item (1) has been evaluated by Xu et al. (2015); the values of (5) and (7) are given error-free because the regions adjacent to voids have an atomistic resolution in our model; item (8) is naturally incorporated because the element boundaries in the coarse-grained domain are assumed to correspond to actual atomic sites. Below, we validate quantities (2), (3), (4), and (6) in CAC and give details of our FR source model. Embedded-atom method (EAM) potentials of Mishin et al. $(1999,2001)$ are adopted because the evaluated GSFE are close to experimentally measured values (Chassagne et al., 2011). In the coarse-grained domain, the post-processing is performed after the atomic positions are interpolated from the nodal positions. Simulation results are visualised using Paraview (Schroeder et al., 2006) and OVITO (Stukowski, 2010). Some runs are completed using Comet and Bridges on the NSF Extreme Science and Engineering Discovery Environment (XSEDE) (Towns et al., 2014).

\subsection{Method validation}

A single dislocation of pure edge or pure screw character is constructed by introducing a Volterra displacement, followed by dynamic simulations at several descending temperatures from $300 \mathrm{~K}$ to $10 \mathrm{~K}$ and quasistatic relaxation (Hartley and Mishin, 2005). The model size is about $102 \mathrm{~nm}$ by $101 \mathrm{~nm}$ by $10 \mathrm{~nm}$, with periodic boundary conditions (PBCs) applied on the last dimension (i.e., the dislocation line direction) while other boundaries are assumed traction free. In the coarse-grained domain, a uniform element size with cases varying from 125 to 4913 atoms per element is adopted. We emphasize that a different model will be introduced for the FR source process in Section 2.2. In all cases, a full dislocation is dissociated into two Shockley partials, the equilibrium distance between them, $w_{\mathrm{SF}}$, is the stacking fault width (Hull and Bacon, 2011) defined 


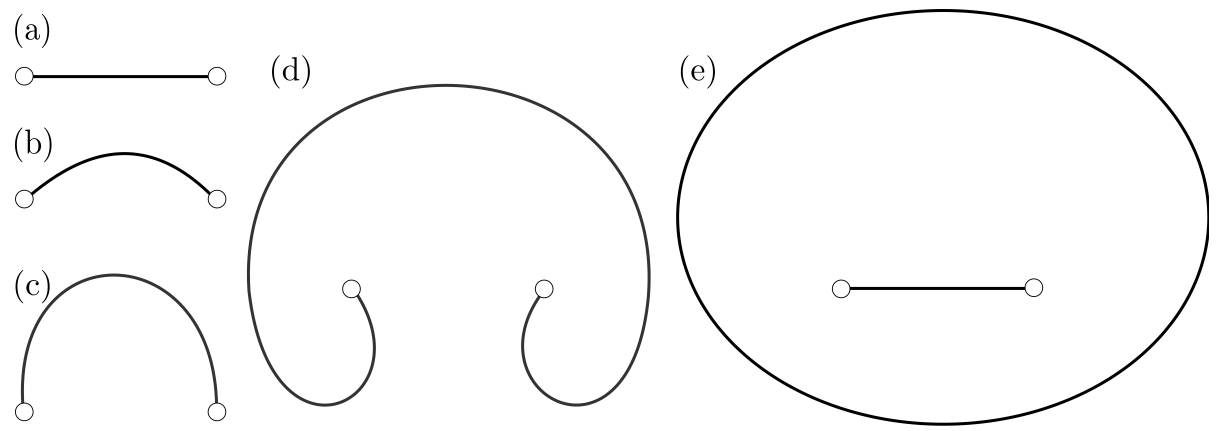

Figure 1: Illustrations of the FR source process. (a) A straight dislocation line is pinned between two voids. (b) The dislocation begins to bow out subject to a shear stress applied on the gliding plane along the upward direction. (c) The dislocation reaches its critical semi-elliptic shape. (d) If the applied stress is larger than the critical shear stress, the dislocation will pass the critical point and continue bowing-out. (e) Two parts of the "kidney shaped" bowed out segments collide to form a dislocation loop, leaving behind a new, straight dislocation line pinned between two voids. Note that both ends of the dislocation move around the void periphery.

as the distance between two points with maximum shear stress magnitude, as shown in Fig. 2. To estimate the full dislocation core radius $r_{0}$, we calculate the strain energy per unit length $E_{\text {strain }}$ of a straight dislocation with length $L_{\mathrm{d}}$ by subtracting cohesive energy from the total energy of a cylinder with a radius $r$ with the dislocation along its centroid axis, i.e.,

$$
E_{\text {strain }}(r)=\frac{1}{L_{\mathrm{d}}}\left[E_{\text {total }}(r)-N E_{\text {coh }}\right]
$$

where $N$ is the number of atoms within the cylinder and $E_{\text {coh }}$ is the cohesive energy. Following Osetsky and Bacon (2003), we define $r_{0}$ as the cylinder radius beyond which $E_{\text {strain }}(r)$ increases linearly with $\ln r$, and so the core energy $E_{\text {core }}=E_{\text {strain }}\left(r_{0}\right)$. Note that $r_{0}$ can be any arbitrary value (Li et al., 2004), yet our goal in this work is to examine if the coarse-grained and atomistic domains give similar $r_{0}$ and $E_{\text {core }}$ for the same geometry under the same boundary conditions; a comparison of $r_{0}$ and $E_{\text {core }}$ between different materials is not important.

The Peierls stress $\sigma_{\mathrm{P}}$ is defined as the minimum stress required to translate a dislocation at $0 \mathrm{~K}$ (Lubarda and Markenscoff, 2007). Following the method of 

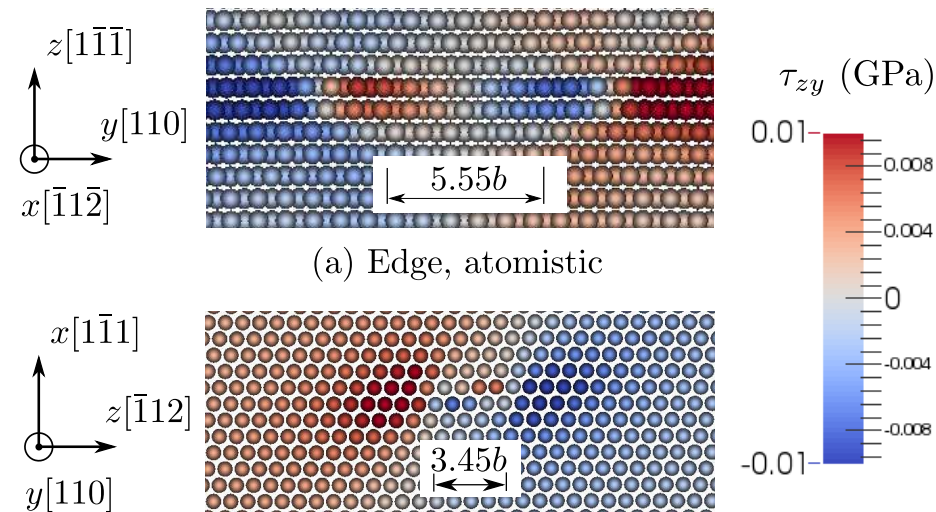

(b) Screw, atomistic

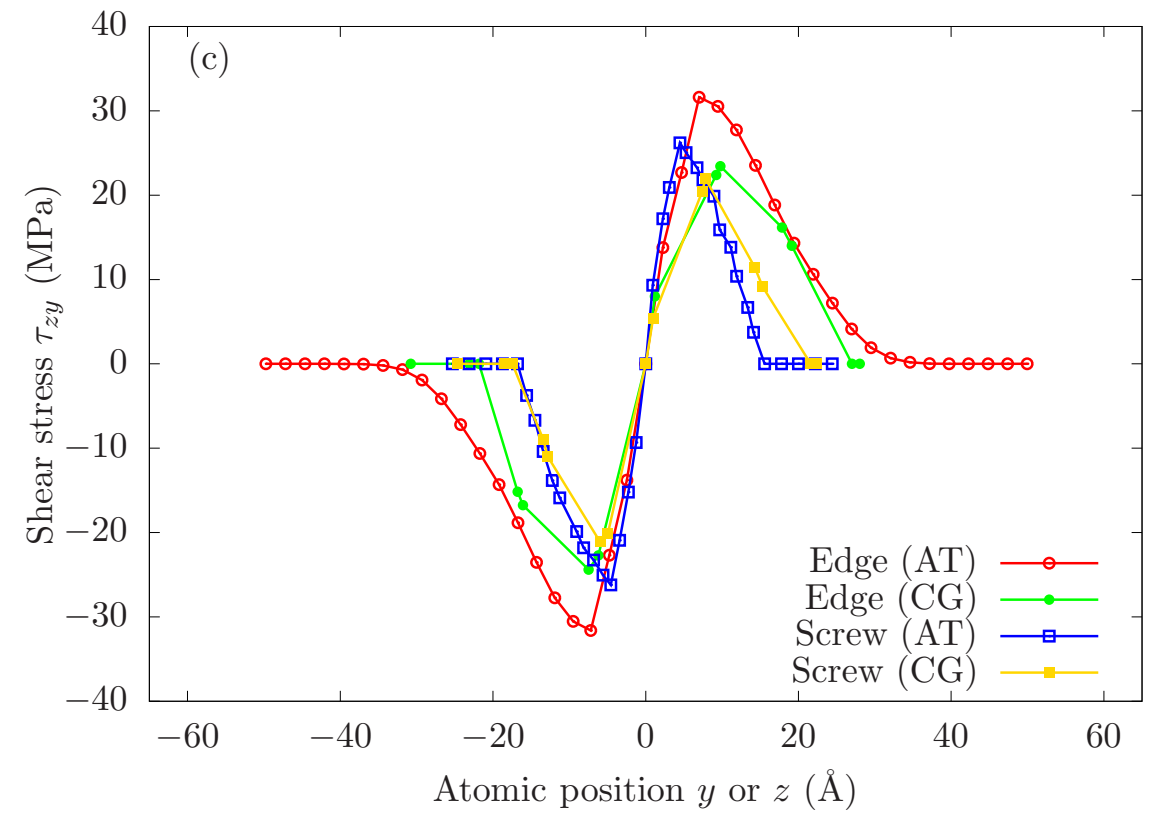

Figure 2: Snapshots of the shear stress fields around a single dislocation with (a) pure edge and (b) pure screw character in the atomistic domain for $\mathrm{Cu}$. PBCs are applied along the dislocation line direction, i.e., the $x$ axis in (a) and the $y$ axis in (b). These models are employed to calculate the stacking fault width, full dislocation core radius, Peierls stress, and dislocation core energy. The shear stress $\tau_{z y}$ profiles of a single dislocation along the $y$ (edge) or the $z$ (screw) direction in both atomistic (AT) and coarse-grained (CG) domains are given in (c). The stacking fault width, which is defined as the distance between two points with maximum shear stress magnitude, is larger in the coarse-grained domain than in the atomistic domain. 
Srinivasan et al. (2005), we conduct quasistatic simulations with an increment of shear stress $\Delta \tau_{z y}=1 \mathrm{MPa}$ for $\langle 110\rangle\{111\}$ slip system, until both partial dislocations suddenly jump to next valley positions, corresponding to a stress $\tau_{z y}^{\prime}$. Then $\sigma_{\mathrm{P}}=\tau_{z y}^{\prime}-\Delta \tau_{z y} / 2$.

Stress concentrations at holes reduce the critical shear stress for an FR source (Crone et al., 2015). In this work, we apply an external shear stress of 11.36 $\mathrm{MPa}$ and compare the stress profiles around holes in both CAC and full atomistic models.

\subsection{Frank-Read source model}

The simulation cell containing a pair of cylindrical holes extruding through the specimen along the $z$ direction is shown in Fig. 3(a), similar to those employed in our earlier work (Xu et al., 2016b,a). Full atomistic resolution is applied in the vicinity of holes such that the hole surface is more than $2 \mathrm{~nm}$ from the atomistic/coarse-grained interface. Away from the holes, rhombohedral elements, as shown in Fig. 1 of the work of Xiong et al. (2011), are employed in the coarse-grained domain. These 3D discontinuous elements have surfaces corresponding to $\{111\}$ slip planes to accommodate dislocations and intrinsic stacking faults. Within each second nearest neighbor (2NN) element, piecewise continuous first order shape and interpolation functions are used; between elements, neither displacement continuity nor interelement compatibility is required (Xu et al., 2015). Unless indicated otherwise, a uniform element size of 2197 atoms solved by first order Gaussian quadrature is employed to achieve a balance between high accuracy and high efficiency (Xu et al., 2016b); in DD simulations, elements sizes on the order of $b$ are required for the FR process to achieve converged results (Crone et al., 2015). Approximations employed in $\mathrm{CAC}$ simulations are evaluated in this paper and our earlier work (Xu et al., 2015, 2016c; Xiong et al., 2011, 2012a). The lattice orientations are $x[\overline{1} 1 \overline{2}]$, $y[110]$, and $z[1 \overline{1} \overline{1}]$. All boundaries are assumed traction free to alleviate spurious image forces, which are evaluated quantitatively in Appendix A. The model size for three materials differs only by their lattice parameter $a_{0}$, which is 3.615 
$\AA$ for $\mathrm{Cu}, 3.52 \AA$ for $\mathrm{Ni}$, and $4.05 \AA$ for $\mathrm{Al}$. For $\mathrm{Cu}$, the simulation cell has a size of $268.3 \mathrm{~nm}$ by $265.3 \mathrm{~nm}$ by $40.6 \mathrm{~nm}$; in all simulations, the hole diameter $D=2.51 \mathrm{~nm}$, while the interhole ligament distance $L$ varies from $5.01 \mathrm{~nm}$ to $102.68 \mathrm{~nm}$, a range comparable to precipitate spacings in many alloys (Szajewski et al., 2015). As a result, with the smallest $L$, the model has 108,163 elements and 1,200,322 atoms, with 2,065,626 degrees of freedom in total compared with otherwise $238,834,433$ in an equivalent full atomistic model.

A straight edge dislocation with length $L$ is introduced on the mid plane normal to the $z$ axis by moving atoms/nodes inside the solid green lines by Burgers vector $\mathbf{b}=\left(a_{0} / 2\right)[110]$, as illustrated in Fig. 3(a), followed by energy minimization. This is equivalent to introducing an extra plane of atoms in between the two holes on the $x-z$ plane without moving any atoms/nodes. In this way, only one dislocation segment is created and pinned between two cylindrical holes without side arms. Once the equilibrium atomic configuration of the FR source is achieved, a homogeneous shear stress $\tau_{z y}$ is applied - we note that a symmetric stress favors the Orowan mechanism over the Hirsch mechanism (Hatano, 2006). To exclude dislocation cross-slip near the hole surface (Hatano et al., 2008) which doesn't accompany the classical FR process, atoms in adjacent to the holes are constrained within the $x-y$ plane. A dynamic CAC simulation (Appendix B) is performed to show that this model can indeed describe the FR source process illustrated in Fig. 1.

To calculate the critical shear stress, which is difficult to obtain (Zhang et al., 2011, 2012), we employ a method similar to the one for the Peierls stress, i.e., quasistatic simulations with an increment of shear stress $\Delta \tau_{z y}=1 \mathrm{MPa}$ are conducted. Let the minimum stress to form the dislocation loop be $\tau_{z y}^{\prime \prime}$, the critical shear stress for the FR source $\tau_{\mathrm{CAC}}=\tau_{z y}^{\prime \prime}-\Delta \tau_{z y} / 2$. The dislocation bow-out segment at the end of an energy minimization subject to a stress of $\left(\tau_{z y}^{\prime \prime}-\Delta \tau_{z y}\right)$ is recorded as the critical configuration, similar to the method used by Crone et al. (2015). The quasistatic simulations ensure that our results are comparable with elastostatic continuum models. 

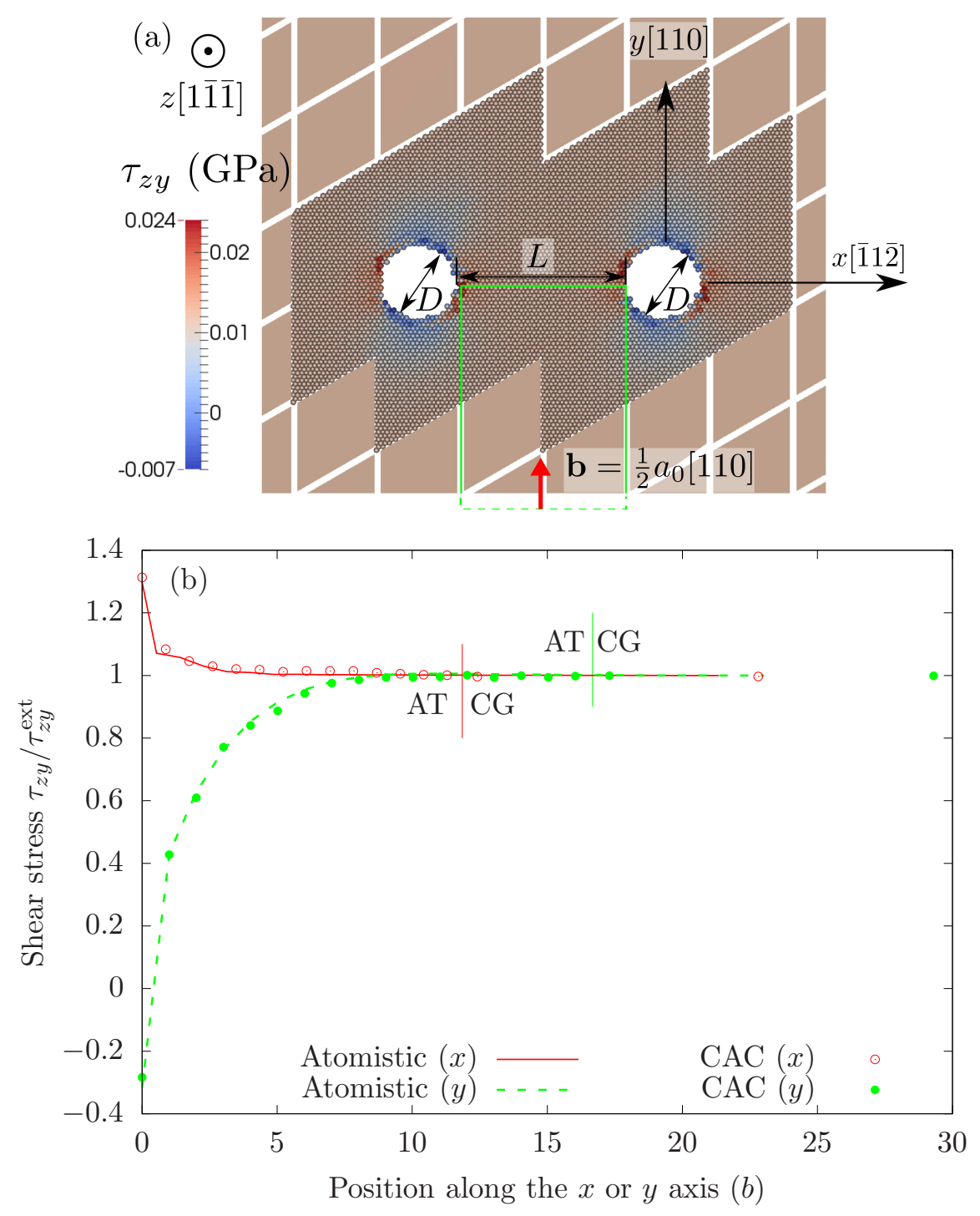

Figure 3: (a) CAC simulation cell of an edge dislocation bowing-out between a pair of cylindrical holes throughout the specimen along the $z$ direction introduced as an FR source. An atomistic (AT) domain is meshed in the vicinity of the holes, while the coarse-grained (CG) domain is employed elsewhere. An external shear stress of $11.36 \mathrm{MPa}$ is applied; atoms/elements are colored by shear stress $\tau_{z y}$. All boundaries are assumed traction free to alleviate spurious image effects. The edge dislocation is formed by moving atoms/nodes inside the solid green lines by Burgers vector $\mathbf{b}$. (b) The ratio of $\tau_{z y}$ relative to the far field external loading $\tau_{z y}^{\text {ext }}=11.36 \mathrm{MPa}$ as a function of the distance from the hole surface along the $x$ and $y$ directions labelled in (a). Stress concentrations around holes are captured correctly in CAC, compared with an equivalent full atomistic model of the same size. 


\section{Results and discussion}

\subsection{Important quantities for the Frank-Read source process}

Values of stacking fault width $w_{\mathrm{SF}}$, full dislocation core radius $r_{0}$, Peierls stress $\sigma_{\mathrm{P}}$, and dislocation core energy per unit length $E_{\text {core }}$ of pure edge and pure screw dislocations in both atomistic and coarse-grained domains for three FCC metals are given in Table 1 . As expected, $r_{0}$ is larger than half of $w_{\mathrm{SF}}$ in all cases, because the energy function outside $r_{0}$ is elastic. Variations of the strain energy $E_{\text {strain }}$ as a function of the cylinder radius $r$ for Ni, calculated by Eq. 5 , are given in Fig. 4(a). It is found that while the coarse-grained domain gives a higher strain energy $E_{\text {strain }}$ near the dislocation core than the atomistic domain, $E_{\text {strain }}$ increases linearly with $\ln r$ beyond $r_{0}$ with the same slope for the same type of dislocation in both domains. Moreover, both domains give the same trend that the edge dislocation has a higher $E_{\text {strain }}$ than the screw dislocation. Figure 4(b) show that in $\mathrm{Ni}$, the coarse-grained domain has a larger $r_{0}$ and $E_{\text {core }}$ than the atomistic domain; both quantities converge to the atomistics as the elements become smaller and contain fewer atoms.

It is found that, within a reasonable range, the coarse-grained domain in CAC predicts a larger $w_{\mathrm{SF}}$, a larger $r_{0}$, a lower $\sigma_{\mathrm{P}}$, and a higher $E_{\text {core }}$, compared with fully resolved atomistics; these are expected because of the flattened, spread out dislocation core structure in the coarse-grained domain along element boundaries (Xu et al., 2015; Cai et al., 2005). In particular, as $E_{\text {core }}$ is positively correlated with the dislocation mobility (Lee et al., 2011), the coarse-grained domain is expected to have a larger dislocation mobility, in agreement with our early work (Xiong et al., 2016). Moreover, both atomistic and coarse-grained domains give the same trend that a screw dislocation has a smaller $w_{\mathrm{SF}}$, a smaller $r_{0}$, a higher $\sigma_{\mathrm{P}}$, and a lower $E_{\text {core }}$, than an edge dislocation. Stress concentrations at holes are captured correctly in CAC, as shown in Fig. 3(b), which indicates that $\mathrm{CAC}$ not only gives an accurate description of the stresses around the holes, but also ensures continuous stress fields across the atomistic/coarsegrained domain interface. 


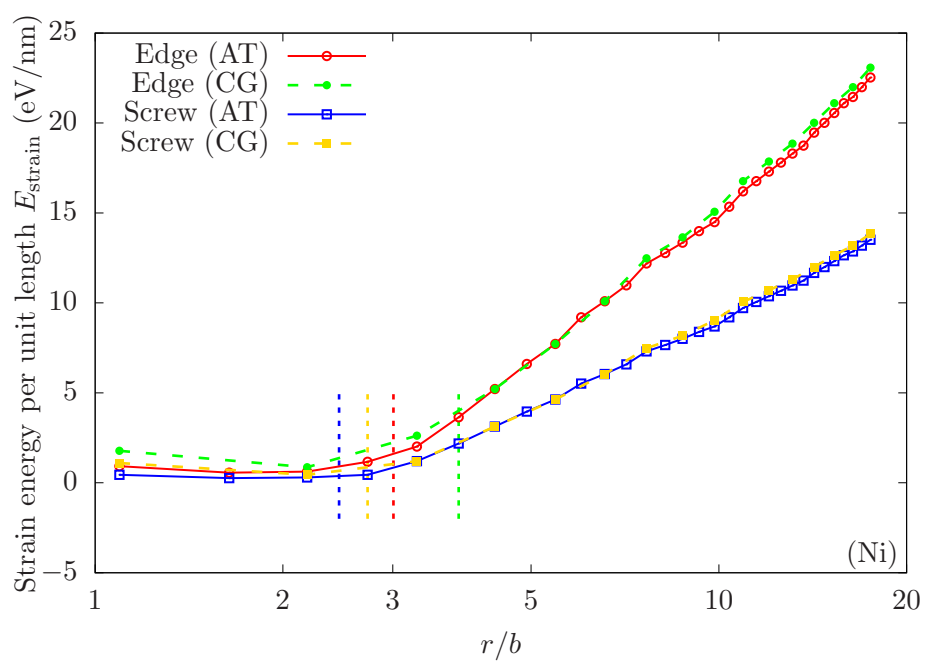

(a)

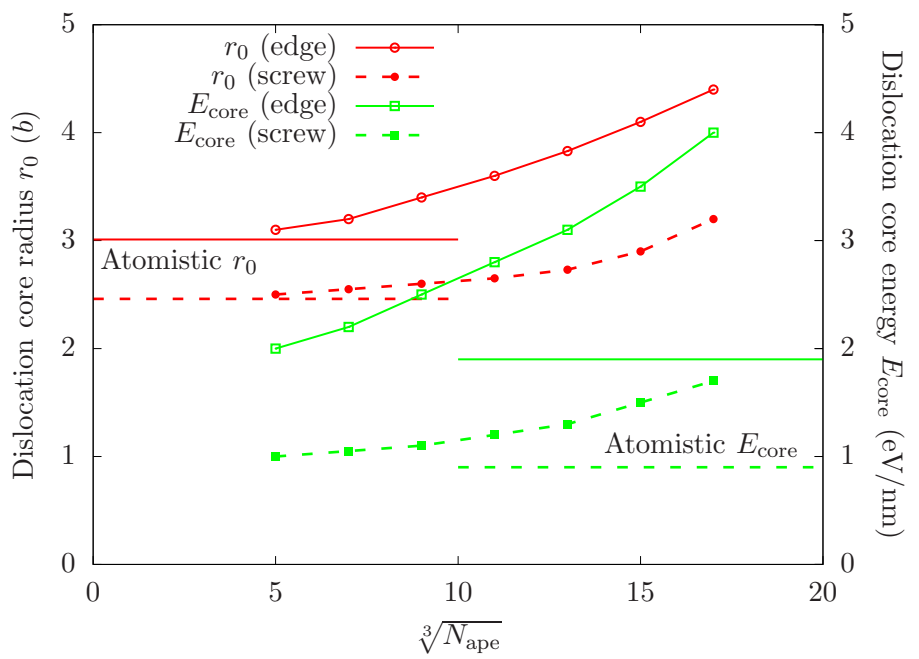

(b)

Figure 4: (a) Strain energy per unit length as a function of cylinder radius $r$ for Ni in both atomistic (AT) and coarse-grained (CG) domains, calculated by Eq. 5. The vertical lines represent the radius $r=r_{0}$ beyond which the strain energy increases linearly with $\ln r$, where $r_{0}$ is taken as the core radius. (b) In $\mathrm{Ni}$, the coarse-grained domain has a larger core radius $r_{0}$ and core energy $E_{\text {core }}=E_{\text {strain }}\left(r_{0}\right)$ than the atomistic domain; both quantities converge to the atomistics (horizontal lines) as each element has a smaller number of atoms $N_{\text {ape }}$. 


\begin{tabular}{ccccccc}
\hline & \multicolumn{2}{c}{$\mathrm{Cu}$} & \multicolumn{2}{c}{$\mathrm{Ni}$} & \multicolumn{2}{c}{$\mathrm{Al}$} \\
& Edge & Screw & Edge & Screw & Edge & Screw \\
\hline$w_{\mathrm{SF}}(\mathrm{AT})$ & 5.55 & 3.45 & 4.71 & 3.02 & 2.60 & 1.42 \\
$w_{\mathrm{SF}}(\mathrm{CG})$ & 6.96 & 4.47 & 5.85 & 4.08 & 3.04 & 1.97 \\
$r_{0}(\mathrm{AT})$ & 4.97 & 2.32 & 3.01 & 2.46 & 1.97 & 1.16 \\
$r_{0}(\mathrm{CG})$ & 5.52 & 2.64 & 3.83 & 2.73 & 2.35 & 1.47 \\
$\sigma_{\mathrm{P}}(\mathrm{AT})$ & 2.50 & 62.50 & 64.50 & 441.50 & 16.50 & 78.50 \\
$\sigma_{\mathrm{P}}(\mathrm{CG})$ & 1.50 & 45.50 & 59.50 & 374.50 & 14.50 & 58.50 \\
$E_{\text {core }}(\mathrm{AT})$ & 1.7 & 1.2 & 1.9 & 0.9 & 2 & 1.6 \\
$E_{\text {core }}(\mathrm{CG})$ & 2.4 & 1.9 & 3.1 & 1.3 & 2.5 & 2.2 \\
\hline
\end{tabular}

Table 1: Stacking fault width $w_{\mathrm{SF}}$ (in $b$ ), full dislocation core radius $r_{0}$ (in $b$ ), Peierls stress $\sigma_{\mathrm{P}}$ (in $\mathrm{MPa}$ ), and dislocation core energy $E_{\text {core }}$ (in $\mathrm{eV} / \mathrm{nm}$ ) for pure edge and pure screw dislocations in both atomistic $(\mathrm{AT})$ and coarse-grained $(\mathrm{CG})$ domains for $\mathrm{Cu}, \mathrm{Ni}$, and $\mathrm{Al}$. In each domain/material, $E_{\text {core }}=E_{\text {strain }}\left(r_{0}\right)$ following Eq. 5 , with $r_{0}$ being its respective core radius.

We remark that despite systematic increase of coarse-graining error in certain quantities relative to fully resolved atomistics with increase of element size, the CAC method allows parametric studies of models at much larger scales with the same computational burden. The elasticity theory upon which the DD method is established considers that the stress field of one dislocation at a long distance is mainly determined by its Burgers vector and character angle. In $\mathrm{CAC}$, although a wider dislocation core is given, the overall Burgers vector and the character angle of a dislocation are preserved, as shown in Fig. 2. Thus, long range stress fields of dislocations are accurately described in CAC. Furthermore, within the same framework, dissociation of partial dislocations and dislocation interactions using $\mathrm{CAC}$ can be accurately described for short range interactions simply by refining the element size. In practice, coarse graining in $\mathrm{CAC}$ is only employed to capture the longer range structures/fields in regions remote from atomistically resolved reactions of interest, so its coarse-graining approximation of details of the dislocation core does not greatly impact the 
accuracy of computed interactions near the pinning points which is critical to the modeling of the FR process.

\subsection{Critical shear stress}

We first perform quasistatic CAC simulations without constraining any atoms in adjacent to the holes, to examine if the constraints influence the critical shear stress. It is found that the screw components of the bowed-out dislocation cross slip in energy minimization because the resolved shear stress is large enough for the athermal limit of cross-slip to be approached. The same phenomenon was also reported in MD simulations (Hatano et al., 2008; Shimokawa and Kitada, 2014). As a result, the critical stress is increased by about $15 \%$, compared with the case without cross-slip. Unlike in MD, dislocation climb is not observed as it usually involves atomic diffusion which can not occur at $0 \mathrm{~K}$. Thus, we remark that while the constraints might influence the critical stress, we can't obtain meaningful results without them because such simulations don't satisfy the classical assumption regarding Orowan bypass made in most continuum models. As the constraints are only applied on atoms near the holes, they do not affect coarse-graining error in any way. In addition, we analytically calculate the image stresses on the dislocation half-loop with the largest $L$ from all traction free boundaries in all three materials, as shown in Appendix A. The image stresses are greatly reduced for smaller $L$ and are not element size-dependent, i.e., they are not the source of the differences between CAC and fully-resolved atomistic simulations.

Eqs. 2-4 contain only one adjustable parameter, namely, the full dislocation core radius $r_{0}$, which is introduced to avoid singularities in the stress field and elastic energy of a dislocation. The dislocation core energy $E_{\text {core }}$ associated with $r_{0}$ is not explicitly included because it is assumed to be of the order of one-tenth of the total energy (Foreman, 1967). In estimating the line tension of a curved dislocation, $E_{\text {core }}$ becomes non-negligible when $L<120$ nm (Szajewski et al., $2015)$. For the FR source, the neglect of $E_{\text {core }}$ leads to an underestimation of the critical shear stress $\tau_{\mathrm{c}}$. A common practice is to deliberately use a small $r_{0}$ 
such that the total energy of a dislocation matches that in atomistic simulations (Li et al., 2004). However, the ambiguities caused by different choices of $r_{0}$ and $E_{\text {core }}$ may affect the calculation of the dislocation energy (LeSar, 2004; Lothe and Hirth, 2005). In some recent DD simulations, $E_{\text {core }}$ was explicitly added to the elastic energy (Shishvan et al., 2008; Martínez et al., 2008), in which its estimation, beyond the scope of elasticity theory (Bacon et al., 1973), is based on $a b$ initio and atomistic calculations, with $r_{0}$ on the order of the Burgers vector of a full dislocation (Cai et al., 2001; Martínez et al., 2008).

Along this line of thought, we modify Eq. 4 by explicitly including $E_{\text {core }}$. First, according to the line tension model (Hull and Bacon, 2011),

$$
\tau_{\mathrm{c}}=\frac{T}{b R}=\frac{\alpha \mu b}{R}
$$

where $T$ is the line tension, $R$ is the dislocation line radius, and $\alpha$ is a parameter. Comparing Eq. 6 to Eq. 4, one sees that

$$
\begin{aligned}
\alpha & =\frac{A}{4 \pi} \ln \left(\frac{\bar{D}}{r_{0}}\right) \\
R & =\frac{L}{2}+\frac{D}{4}
\end{aligned}
$$

We then augment the line tension $T$ by the core energy $E_{\text {core }}$. For an edge FR source, Eq. 6 becomes

$$
\tau_{\mathrm{c}}=\frac{T+C_{0}\left(r_{0} ; E_{\text {core }}\right)}{b R}=\frac{\alpha \mu b^{2}+C_{0}\left(r_{0} ; E_{\text {core }}\right)}{b R}
$$

where, to a first order approximation (Szajewski et al., 2015), we assign

$$
C_{0}\left(r_{0} ; E_{\text {core }}\right)=\frac{1-2 \nu}{1-\nu} \cdot E_{\text {core }}\left(r_{0}\right)
$$

Substituting Eqs. 7, 8, and 10 into Eq. 9 yields

$$
\tau_{\mathrm{c}}=\frac{A \mu b}{2 \pi(L+D / 2)}\left[\ln \left(\frac{\bar{D}}{r_{0}}\right)+\frac{1-2 \nu}{1-\nu} \cdot \frac{4 \pi E_{\text {core }}\left(r_{0}\right)}{\mu b^{2}}\right]
$$

where the last term can be viewed as the material dependent parameter $B$ in Eqs. 2 and 3.

Eqs. 1-4 and 11 are represented in Figs. 5(a), 5(b), and 6(a) for $\mathrm{Cu}, \mathrm{Ni}$, and $\mathrm{Al}$, respectively, as a function of the FR source length. In Eqs. 2-4, the core 


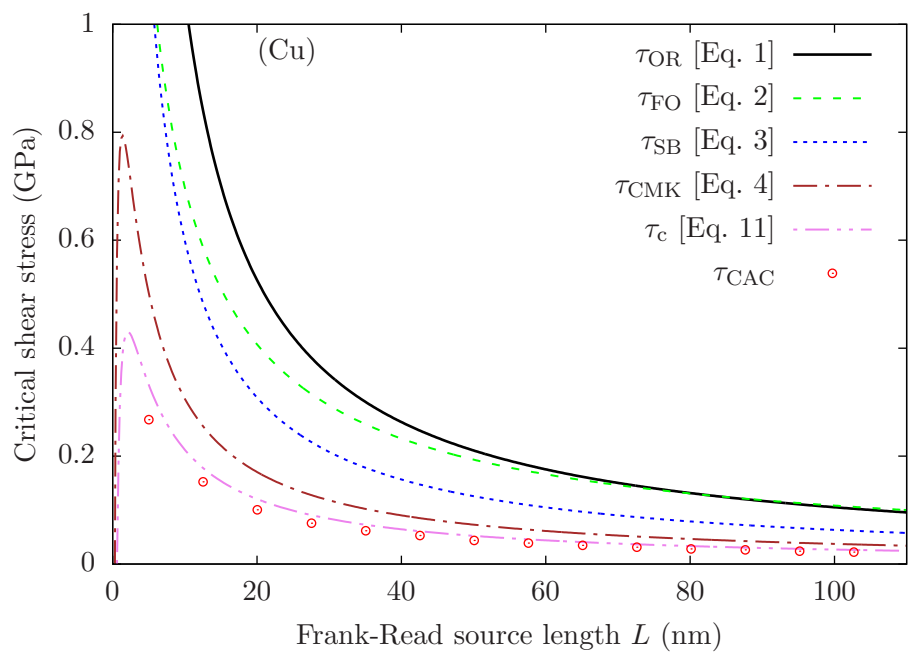

(a)

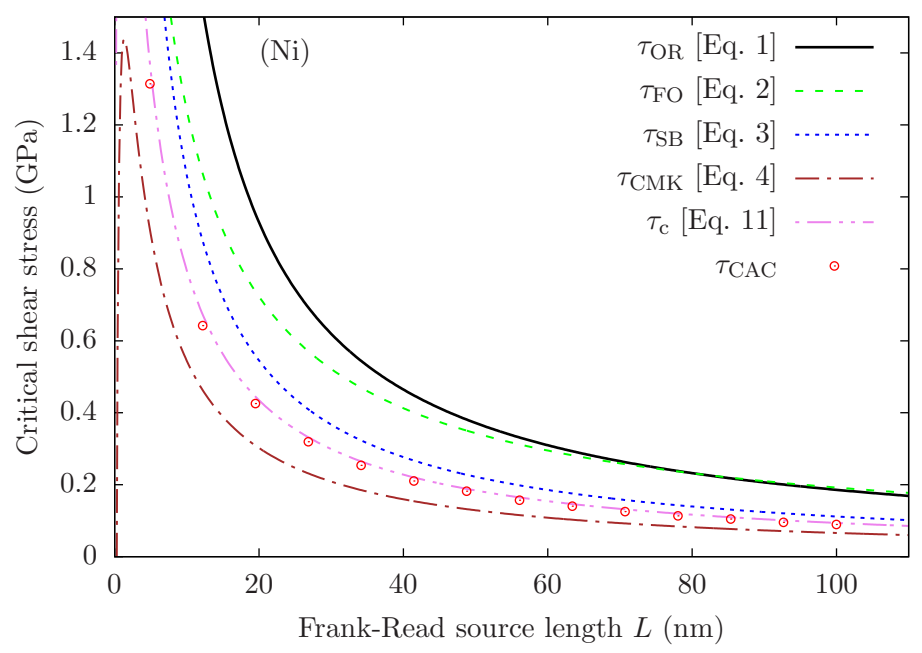

(b)

Figure 5: The critical shear stress predicted by continuum models and CAC simulations with respect to the FR source length in (a) $\mathrm{Cu}$ and (b) $\mathrm{Ni}$. 


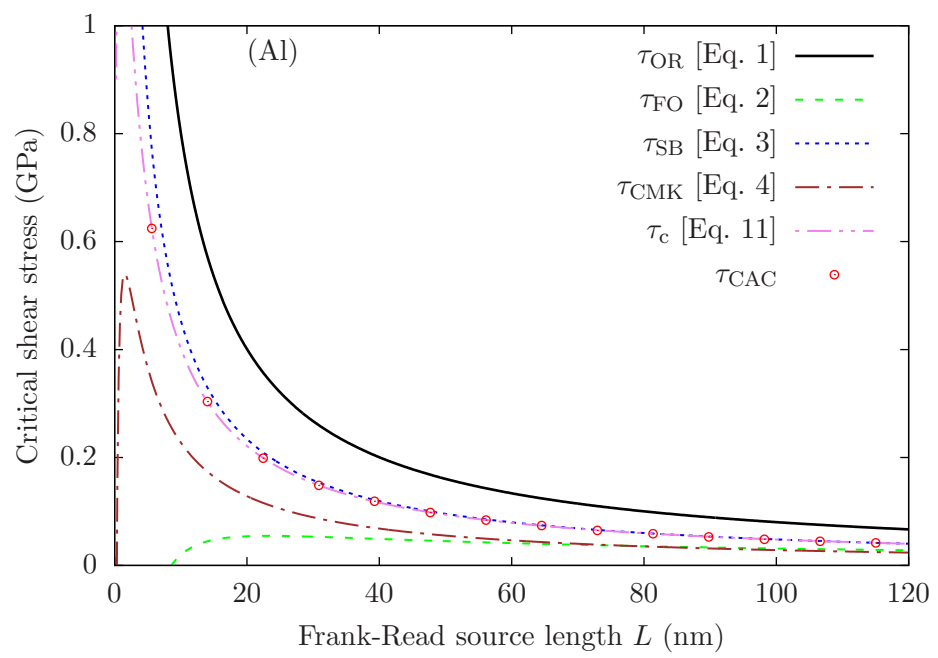

(a)

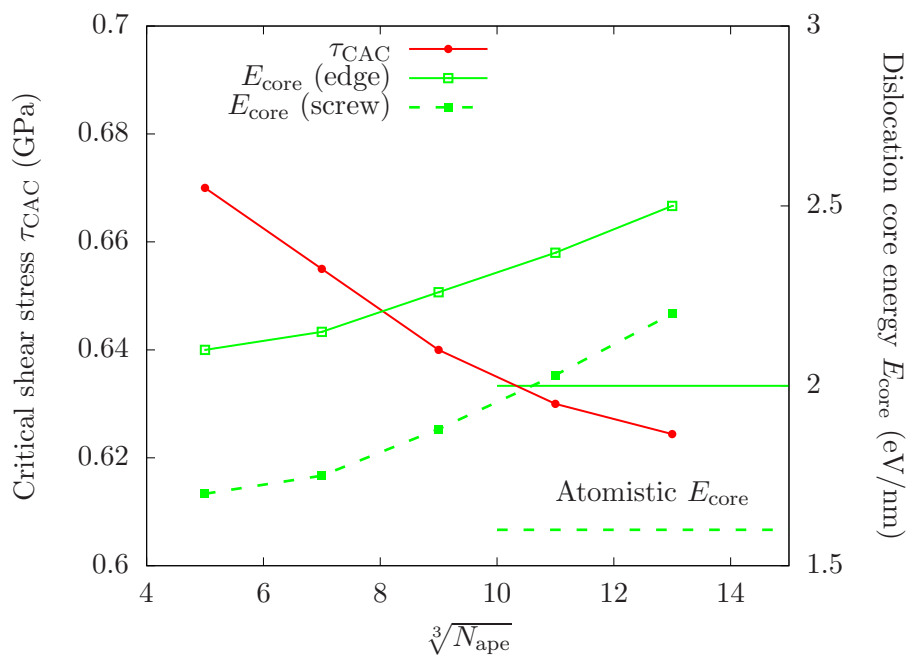

(b)

Figure 6: (a) The same plot as in Fig. 5, but for Al. (b) The critical shear stress $\tau_{\mathrm{CAC}}$ and dislocation core energy $E_{\text {core }}$ for both types of dislocation, as a function of the element size $N_{\text {ape }}$ for $\mathrm{Al}$ in the case of $L=5.61 \mathrm{~nm}$. Larger elements give a lower critical shear stress and higher dislocation core energy for both types of dislocation. In particular, $E_{\text {core }}$ converges to the atomistics (horizontal lines) as each element has a smaller number of atoms $N_{\text {ape }}$. 
radius $r_{0}=b$, as in their original forms. In Eq. 11, $r_{0}$ and $E_{\text {core }}$ are assumed invariant subject to the shear stresses applied in our simulations. Also, because both core radius $r_{0}$ and energy $E_{\text {core }}$ vary along the bowed-out dislocation with mixed characters (Lothe and Hirth, 2005), the line averages of their respective values for pure edge and pure screw dislocations in Table 1 are used, as given in Appendix C. We remark that the values of $r_{0}$ and $E_{\text {core }}$ are taken from the coarse-grained domain, in which the FR source mainly operates. Since both quantities vary with element size, as shown in Fig. 4(b), the critical stress calculated by Eq. 11, $\tau_{\mathrm{c}}$, is also element size-dependent. According to Eq. 11, larger elements result in a larger $r_{0}$, which decreases $\tau_{\mathrm{c}}$, and a larger $E_{\text {core }}$, which increases $\tau_{\mathrm{c}}$ - the net effect is that a lower $\tau_{\mathrm{c}}$ is yielded, in agreement with our CAC simulations for $\mathrm{Al}$, as shown in Fig. 6(b). The combined effect of the element size-dependent $r_{0}$ and $E_{\text {core }}$ also results in a less pronounced dependence of $\tau_{\mathrm{c}}$ on the element size. It is found that the element size- and $L$ dependent coarse-graining error (1) is reduced for a larger $L$, suggesting that the precise value of $E_{\text {core }}$ becomes less important as the FR source length increases, and (2) increases for a smaller $L$, yet the majority of the dislocation half-loop would reside in the atomistic domain because the domains adjacent to the holes are modeled with full atomistic resolution. We emphasize that the form of Eq. 11, suggested by our coarse-grained simulations is most relevant to informing higher scale continuum models such as crystal plasticity finite element method (CPFEM) or certain DD models that involve constitutive equations for FR source generation (Balint et al., 2008; Deshpande et al., 2005). In such relations, $a b$ initio and atomistic values of $r_{0}$ and $E_{\text {core }}$ should be used.

For application purposes, the scaling of the critical stress with respect to the FR source length $L$ is more important than the critical stress itself and can be used to obtain the dislocation core energy or line tension for a given material system. For all three materials, the critical stress calculated by the CAC simulations, $\tau_{\mathrm{CAC}}$, scales with $L$ logarithmically, as predicted by the continuum models. In terms of the values of $\tau_{\mathrm{CAC}}$ for a given $L$, however, only the critical stresses in $\mathrm{Al}$ agree well with Eq. 11. For $\mathrm{Cu}$ and $\mathrm{Ni}, \tau_{\mathrm{CAC}}$ are 
lower than the continuum predictions, which may be attributed to two simplifications in deriving the model. First, dislocations in $\mathrm{Cu}$ and $\mathrm{Ni}$ have a strong dissociation due to a low SFE. As a result, the total energy of an extended dislocation, which is proportional to $b^{2}$, is smaller than an undissociated one (Bacon, 1967). It follows that the work that needs to be done by the external stress is reduced, a conclusion also reached by a recent DD simulation (Huang et al., 2014). Second, all continuum models described above assume isotropic shear modulus and Poisson's ratio. On the other hand, the anisotropy of a crystal has been known to alter the relative energies/core radius of edge and screw dislocations (Foreman, 1967), as well as the elastic strain around a dislocation which is important for dislocation-void interactions (Hatano and Matsui, $2005)$. For a cubic system, the anisotropy indices $A_{\text {cubic }}=2 c_{44} /\left(c_{11}-c_{12}\right)$ (Ranganathan and Ostoja-Starzewski, 2008), which gives 3.22, 2.53, and 1.21 for $\mathrm{Cu}$, $\mathrm{Ni}$, and $\mathrm{Al}$, respectively, based on EAM potential predicted elastic constants $c$. Fitzgerald (2010) found that the critical shear stress for an FR source decreases with an increasing $A_{\text {cubic }}$. In other words, the isotropic models overestimate the bowing-out stress, especially for $\mathrm{Cu}$ which has the largest elastic anisotropy.

This dependence of model accuracy on materials was also reported for the SB model (Eq. 3). For Al, the SB model accurately predicts the critical shear stress of an FR source in comparison with MD simulations (Shimokawa and Kitada, 2014). For $\mathrm{Cu}$ and Fe, however, Eq. 3 gives a higher depinning stress for dislocation-void interaction than MD (Hatano and Matsui, 2005; Lehtinen et al., 2016). Therefore, the significance of dislocation dissociation and elastic anisotropy suggests reconsideration of isotropic elastic models employed in continuum theory and DD simulations. Although methods have been developed to calculate the energy and force on a dislocation in an anisotropic elastic media (Aubry et al., 2014), we are not aware of any fully anisotropic continuum model for the FR source at this point. 

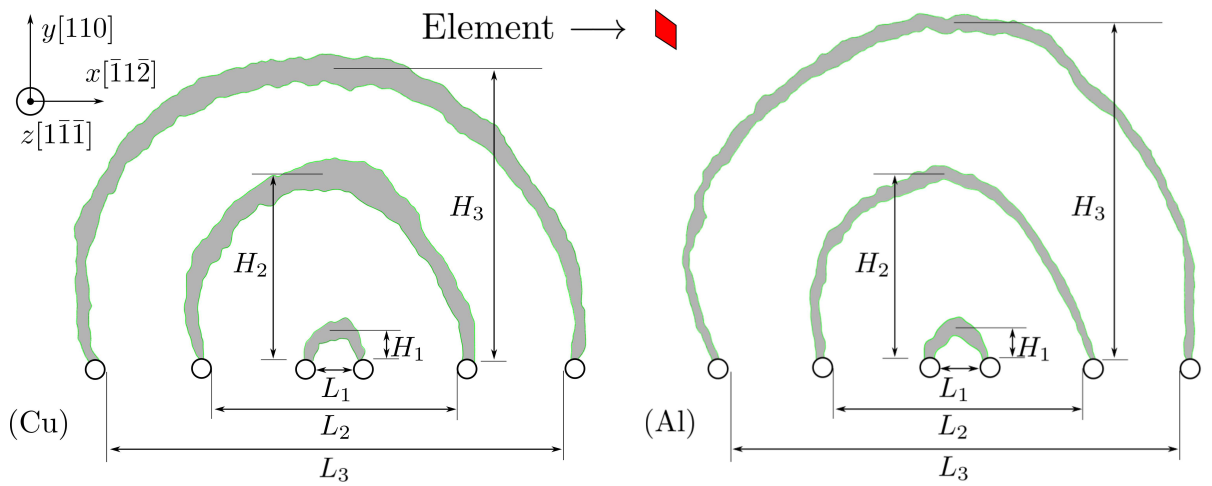

Figure 7: Snapshots of critical dislocation configurations for different FR source length $L$ in $\mathrm{Cu}$ and $\mathrm{Al}$. The green curves represent Shockley partial dislocations identified by a Dislocation Extraction Algorithm (Stukowski et al., 2012); the gray ribbons are intrinsic stacking faults. The kinks along dislocations have a size on the order of that of an element, which is illustrated as a red parallelogram. For $\mathrm{Cu}, L_{1}=5.01 \mathrm{~nm}$ with $H_{1}=0.7 L_{1}, L_{2}=35.07 \mathrm{~nm}$ with $H_{2}=0.77 L_{2}$, and $L_{3}=65.13 \mathrm{~nm}$ with $H_{3}=0.69 L_{3}$. For Al, $L_{1}=5.61 \mathrm{~nm}$ with $H_{1}=0.8 L_{1}$, $L_{2}=39.29 \mathrm{~nm}$ with $H_{2}=0.8 L_{2}$, and $L_{3}=72.97 \mathrm{~nm}$ with $H_{3}=0.75 L_{3}$.

\subsection{Critical dislocation configuration}

The critical dislocation configuration corresponds to an extremum point in the dislocation total energy (Shishvan et al., 2008), the largest dislocation line tension (Szajewski et al., 2015), the moment when the dislocation becomes tangent to the surface in the Orowan mechanism (Crone et al., 2015), and the point after which the number of defect atoms dramatically increases (Shimokawa and Kitada, 2014). In CAC, snapshots of dislocations at the onset of instability are shown in Fig. 7 for $\mathrm{Cu}$ and $\mathrm{Al}$. The segments are nearly semi-elliptic in shape with slight horizontal outward bowing near two holes, as a result of a dislocation inclining to have as large a screw component, which has a smaller elastic energy than the edge component, as possible (Hull and Bacon, 2011; Foreman, 1967; Gurrutxaga-Lerma et al., 2015). In addition, the dislocation half-loop is asymmetric with respect to the $y$ axis due to the anisotropy of the Shockley partial dislocations, which tend to adopt their screw orientations close to holes to reduce the total energy (deWit and Koehler, 1959; Hatano and Matsui, 2005; Huang et al., 2014). Note that an anisotropic elastic theory 


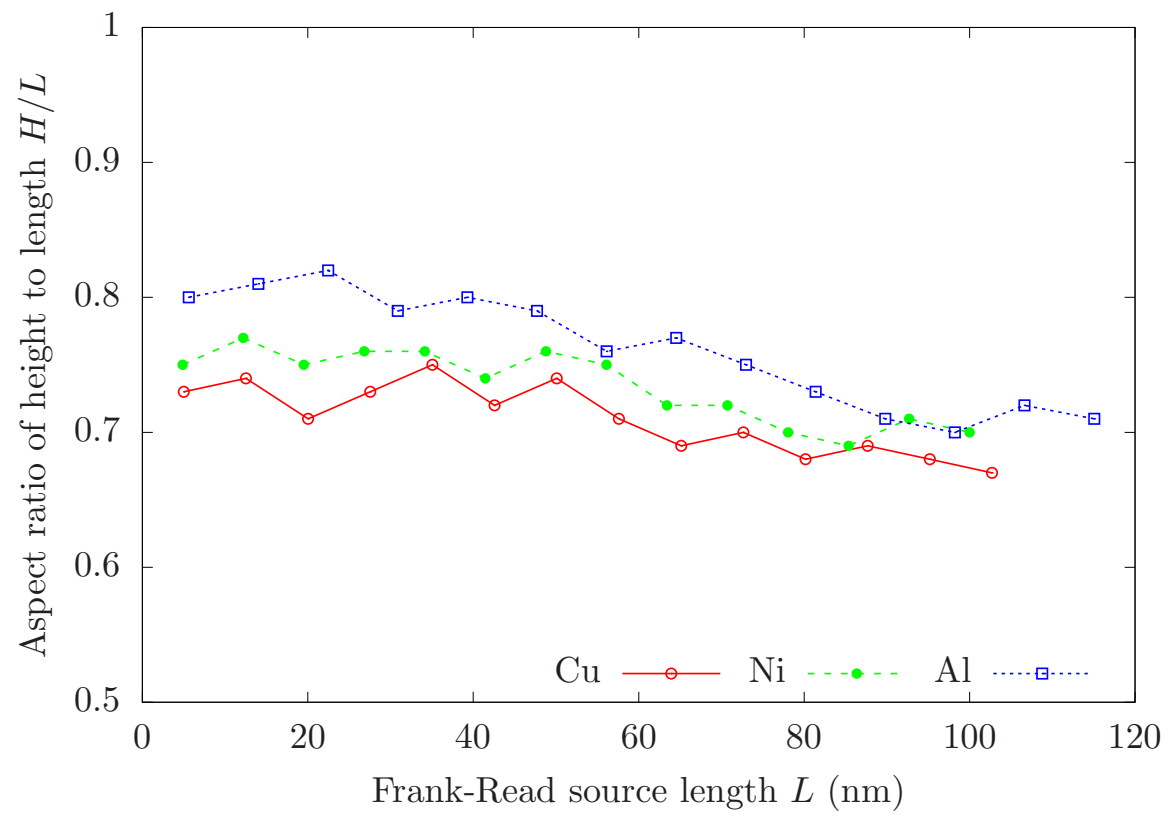

Figure 8: The aspect ratio of the dislocation half-loop height to the FR source length $H / L$, as a function of $L$. For the same $L$, the ratio for $\mathrm{Cu}$ is the smallest while that for $\mathrm{Al}$ is the largest. In general, the ratio $H / L$ decreases with an increasing $L$.

without dislocation dissociation still results in a symmetric shape (Huang et al., 2014). In both materials, the edge components have a larger $w_{\mathrm{SF}}$ than the screw components. Compared with $\mathrm{Al}$, the same dislocation component has a larger $w_{\mathrm{SF}}$ because of a smaller SFE in $\mathrm{Cu}$. We remark that the kinks along dislocations have a size on the order of that of an element, which is illustrated as a red parallelogram in Fig. 7. Clearly, element discretization is a source of coarse-graining error in the dislocation line energy.

The ratio of the dislocation half-loop height $H$ to the FR source length $L$ is plotted in Fig. 8 for three materials. Both the anisotropic elastic theory (deWit and Koehler, 1959) and MD simulations (Shimokawa and Kitada, 2014) predict that $H / L=1 /(2-2 \nu)$. DD simulations of a pointlike FR source using a uniform drag coefficient without dislocation dissociation give an $H$ of $0.83 \mathrm{~L}$ (Bacon, 1967) or $0.75 L$ (Foreman, 1967), independent of L. In contrast, our 
CAC simulations show that the aspect ratio $H / L$ varies between 0.67 and 0.82 for different $L$. The general trend is that a larger $L$ is accompanied by a smaller $H / L$, in agreement with a DD simulation with finite size FR sources (Bacon et al., 1973) or pointlike FR sources with an anisotropic drag coefficient (Bacon, 1967; Gurrutxaga-Lerma et al., 2015). Compared with $\mathrm{Ni}$ and $\mathrm{Al}$, the aspect ratio $H / L$ is smaller in $\mathrm{Cu}$ for a large $L$, suggesting that a larger $w_{\mathrm{SF}}$ reduces $H / L$, a phenomenon also found by Huang et al. (2014). The difference of the critical dislocation configuration in different materials may also be attributed to the material anisotropy, which is found to greatly influence the equilibrium dislocation shape (Fitzgerald et al., 2012).

\section{Conclusions}

In this paper, large scale quasistatic CAC simulations are employed to calculate the critical shear stress and critical dislocation configuration for the process of an edge dislocation segment bowing-out from a classical FR source associated with cylindrical holes as obstacles in $\mathrm{Cu}, \mathrm{Ni}$, and $\mathrm{Al}$. Results are summarized as follows:

1. A number of important quantities for the FR source modelling, including dislocation dissociation, Peierls stress, and dislocation core width/energy, are calculated using both fully resolved atomistic and fully coarse-grained simulations. With decreasing element size, the coarse-grained values converge to those of fully resolved atomistic simulations. In addition, both atomistic and coarse-grained domains give the same magnitude relation in these values of pure edge and pure screw dislocations. Moreover, stress concentrations around holes are captured correctly in CAC simulations with a fully resolved atomistic domain near the holes.

2. It is found that the critical stresses calculated by CAC simulations in all three metals scale with the FR source length in the same way as predicted by a continuum model that explicitly includes the dislocation core energy. The value of the critical stress for a given FR source length, however, only 
agrees well with the continuum model in $\mathrm{Al}$, but not in $\mathrm{Cu}$ and $\mathrm{Ni}$ which have a stronger dislocation dissociation and a higher elastic anisotropy index. The element size-dependent critical stress in CAC is explored in which a larger element yields a lower FR source strength, in agreement with the continuum model. Our CAC simulations suggest reconsideration of isotropic elastic models without dislocation dissociation in deriving continuum theories for the FR source process.

3. CAC simulations show that the critical dislocation segments have an asymmetric semi-elliptic shape, as a result of both dislocation dissociation and material anisotropy. The ratio of the dislocation half-loop height to the FR source length decreases with an increasing FR source length. For the same FR source length, the largest ratio is obtained for $\mathrm{Cu}$ while the smallest for $\mathrm{Al}$.

By taking the FR source operation as a benchmark problem, it is shown that the CAC approach is useful at intermediate length scales between fullyresolved atomistics and DD/PFM/CPFEM. In this regard, CAC can serve as a complement to methods at the lower and higher length scales. Compared with $\mathrm{MD} / \mathrm{MS}$, the advantage of CAC is that with greatly reduced degrees of freedom, the key characteristics of the FR process can be reasonably well described, despite a wider dislocation core and a higher core energy. Hence, it is shown that even with systematic coarse-graining error, as quantified in this work, CAC can provide useful guidance regarding the form of higher scale constitutive models. As the element size is reduced, the CAC predictions approach the fully atomistic results. Compared with DD, in which only the dislocation lines are resolved, CAC simulations contain more degrees of freedom and are less computationally efficient. We remark that the CAC method is especially useful to explore problems in which full atomistic resolution is required in some regions (e.g., complex atomistic phenomena involving dislocations reactions with other defects), with coarse-graining employed elsewhere to support representation of dislocation interactions and transport. In such cases, dislocation lines span between fully 
resolved atomistic and coarse-grained domains. Examples include, but are not limited to, dislocation/GB interactions, dislocation/obstacle interactions, and dislocation substructure evolution.

It is anticipated that our results may assist in formulating constitutive laws and rules that may be useful upstream in PFM, certain DD codes (Balint et al., 2008; Deshpande et al., 2005), and CPFEM for obstacle-induced work hardening. For example, in some CPFEM work (Han et al., 2004), the critical shear stress is incorporated into the isotropic hardening term and the yield surface. FR sources with other initial dislocation character such as pure screw and mixed type, as well as different line orientations and shearing directions, will be assessed in future work.

Under dynamical loading, the work dissipated by the bow-out may not be balanced by dislocation line tension, suggesting reformulation of the continuum models for critical stress discussed in this paper. Moreover, dislocation segments have the lowest mean glide velocity at the critical shape (Benzerga, 2008), and a high strain rate and/or applied shear stress will reduce the aspect ratio $H / L$ (Gurrutxaga-Lerma et al., 2015); the dislocation configuration can be further complicated by that the mobility of an edge component is higher than that of a screw component (Cai et al., 2005) such that the critical configuration at different stress levels will be significantly different (Shishvan et al., 2008), a phenomenon that merits further investigation. Following our earlier CAC simulations (Xiong et al., 2015) which found that a high strain rate reduces the void strengthening effect, it will also be interesting to explore the FR source behavior dependence on the strain rate.

\section{Acknowledgments}

These results are based upon work supported by the National Science Foundation as a collaborative effort between Georgia Tech (CMMI-1232878) and University of Florida (CMMI-1233113). Any opinions, findings, and conclusions or recommendations expressed in this material are those of the authors 
and do not necessarily reflect the views of the National Science Foundation. The work of LX was supported in part by the Department of Energy, Office of Basic Energy Sciences under Award Number DE-SC0006539. The authors thank Dr. Tomotsugu Shimokawa, Dr. Benjamin Szajewski, Dr. Dengke Chen, Dr. Matthew Priddy, Ms. Yanqing Su, Mr. Zhi Zeng, and Mr. Luke Costello for helpful discussions. This work used the Extreme Science and Engineering Discovery Environment (XSEDE), which is supported by National Science Foundation grant number ACI-1053575.

\section{Appendix A. Image stresses}

In the FR source model, all boundaries of the simulation cell as well as the hole surfaces are traction free. The image stresses on the bow-out from all boundaries can be estimated by assuming that all image dislocations are straight (Szajewski and Curtin, 2015). Consequently, the image stresses arising from the surfaces normal to the $x$ axis are zero according to Brown's formula (Brown, 1967). The effective images stress $\tau_{\text {img,eff }}$ from the 2 -D image dislocation array along both the $y$ and $z$ directions are (Szajewski and Curtin, 2015):

$$
\begin{aligned}
\tau_{\text {img,eff }} & =-\frac{4 \mu b H}{45 L_{y}^{2} L_{z}^{2}} \cdot\left[L_{y}^{2} A(\bar{L})+L_{z}^{2} B(\bar{L})\right] \\
A(\bar{L}) & =\frac{2}{\pi(1-\nu)} \int_{0}^{\infty} \frac{n^{3} \tanh ^{2}(n) \sin \left[\left(2 N_{\mathrm{img}}+1\right)(n \bar{L})\right]}{[n-\cosh (n) \sinh (n)] \sin (n \bar{L})} \mathrm{d} n \\
B(\bar{L}) & =\frac{\pi}{1-\nu}\left[\sum_{n=1}^{\infty}(-1)^{n} \operatorname{csch}^{2}\left(\frac{n \pi}{\bar{L}}\right)\left(1-\frac{2 n \pi}{\bar{L}} \operatorname{coth}\left(\frac{n \pi}{\bar{L}}\right)\right)-\frac{1}{6}\right]
\end{aligned}
$$

where $N_{\mathrm{img}}=25 ; L_{y}$ and $L_{z}$ are the length of the simulation cell along the $y$ and $z$ axes, respectively; $\bar{L}=L_{y} / L_{z}=6.5345$. Substituting the values of $\mu$, $b, \nu, L_{y}, L_{z}$, and $H$ at the critical configuration for the largest $L$ into Eq. A.1 yields $\tau_{\text {img,eff }}$ for three materials, as shown in Table $\mathrm{A} .2$. For $\mathrm{Cu}, \mathrm{Ni}$, and $\mathrm{Al}$, the

critical shear stresses for the largest $L$ are $0.044 \mathrm{GPa}, 0.09 \mathrm{GPa}$, and $0.042 \mathrm{GPa}$, respectively. Thus, the relative errors arising from the effective image stresses are $0.00865 / 0.044=0.197,0.01478 / 0.09=0.164$, and $0.00553 / 0.042=0.132$, respectively. Note that (1) this net image shear stress promotes dislocation 
bow-out and (2) the actual image stress in our model is smaller than $\tau_{\text {img,eff }}$ because Eq. A.1 assumes PBCs, resulting in an infinite array of dislocations of the same Burgers vector along the $y$ direction; on the other hand, the traction free boundaries of our simulation cell lead to an infinite array of dislocations of alternating Burgers vector along the $y$ direction, hence reducing the net image stress.

\begin{tabular}{cccccccc}
\hline & $L_{y}$ & $L_{z}$ & $H$ & $\mu$ & $b$ & $\nu$ & $\tau_{\text {img,eff }}$ \\
\hline $\mathrm{Cu}$ & 265.3 & 40.6 & 72.9 & 41.16 & 0.256 & 0.34 & 0.00865 \\
$\mathrm{Ni}$ & 258.33 & 39.53 & 69.99 & 74.67 & 0.249 & 0.31 & 0.01478 \\
$\mathrm{Al}$ & 297.22 & 45.49 & 78.22 & 28 & 0.286 & 0.33 & 0.00553 \\
\hline
\end{tabular}

Table A.2: The length of the simulation cell along the $y$ and $z$ axes $L_{y}$ and $L_{z}$ (in $\mathrm{nm}$ ), dislocation half-loop height for the largest FR source length $H$ (in $\mathrm{nm}$ ), shear modulus $\mu$ (in GPa), magnitude of the Burgers vector of the full dislocation $b$ (in $\mathrm{nm}$ ), Poisson's ratio $\nu$, and the effective image stress $\tau_{\text {img,eff }}$ (in GPa) calculated by Eq. A.1.

\section{Appendix B. Frank-Read source process in dynamic CAC}

A dynamic CAC simulation of the FR source process is performed at $10 \mathrm{~K}$ with time step of $2 \mathrm{fs}$ for $\mathrm{Ni}$. In each run, a constant applied shear stress 1.4 GPa is maintained using a Parrinello-Rahman barostat (Parrinello and Rahman, 1981), resulting in a slightly increasing shear strain due to an increasing plastic shear strain caused by dislocation multiplication. We define the dislocation nucleation time $t_{\text {nuc }}$ as the waiting time for a dislocation segment to reach its critical configuration (Benzerga, 2008) and the loop formation time $t_{\text {loop }}$ as the time for the occurrence of a collision of two parts of the "kidney shaped" bowed out dislocation segments (Shishvan et al., 2008). We remark that in dynamic CAC with an fs-scale time step, the values of $t_{\text {nuc }}$ and $t_{\text {loop }}$ are not directly comparable with those of elastostatic predictions (Hatano and Matsui, $2005)$, yet their ratio $t_{\text {loop }} / t_{\text {nuc }}$ should not be much different from the continuum estimation. As shown in Fig. B.9 for Ni, the critical dislocation configuration is 


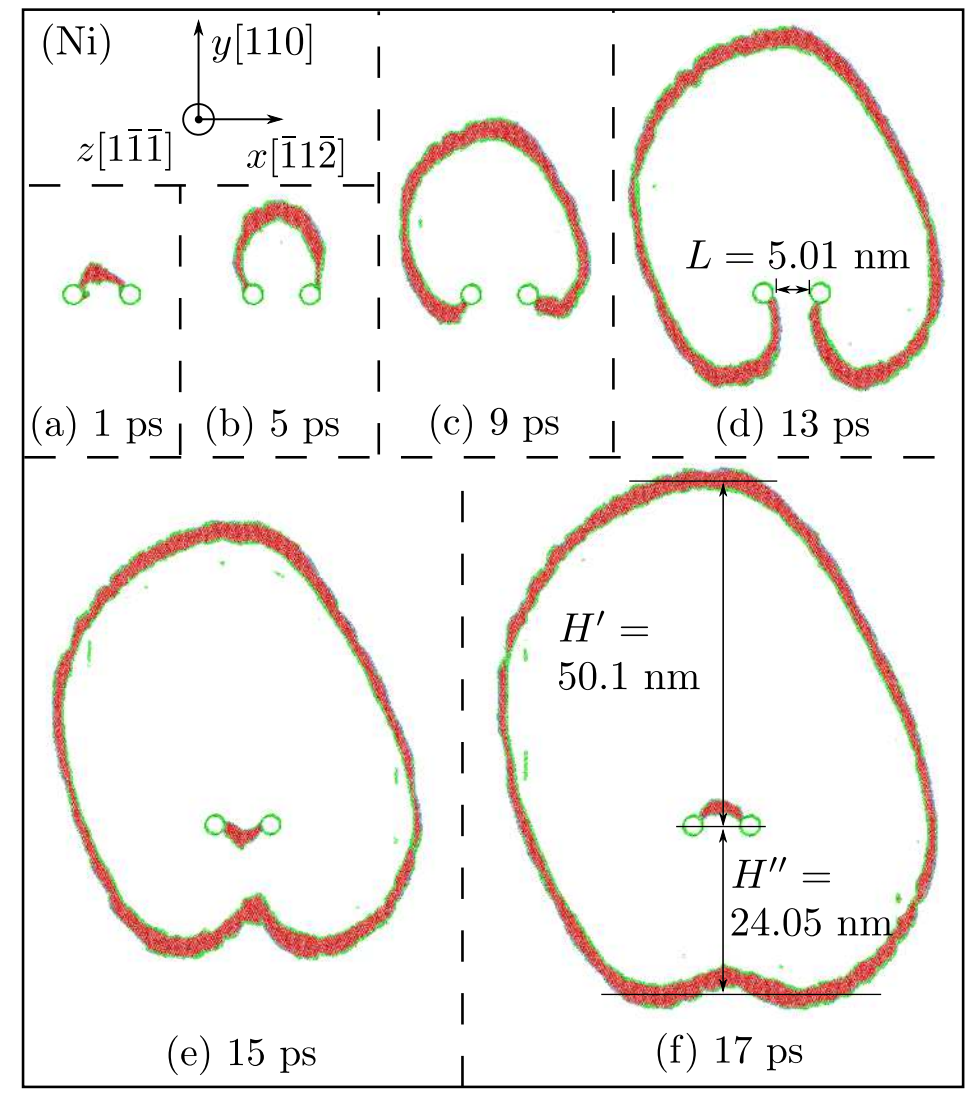

Figure B.9: Snapshots of dislocation loop multiplication from the FR source in Ni subject to a constant applied shear stress of $1.4 \mathrm{GPa}$. Atoms are colored by adaptive common neighbor analysis (Stukowski, 2012): red are of hexagonal close-packed local structure, green are either hole surfaces or Shockley partial dislocations, and all FCC atoms are deleted. In (f), the distance between the top of the loop and holes $\left(H^{\prime}\right)$ is about twice the distance between the bottom of the loop and holes $\left(H^{\prime \prime}\right)$.

reached after 4 ps while the dislocation loop is formed at about $14 \mathrm{ps}$, resulting in $t_{\text {loop }} / t_{\text {nuc }}=3.5$. In comparison, DD simulations (Shishvan et al., 2008) based on an elastostatic theory predict that $t_{\mathrm{loop}} / t_{\mathrm{nuc}} \approx 2.89$ and 2.92 for isotropic and anisotropic drag coefficients, respectively. 


\section{Appendix C. Line averages of $r_{0}$ and $E_{\text {core }}$}

Similar to the drag coefficient in DD simulations (Martínez et al., 2008), we assume both $r_{0}$ and $E_{\text {core }}$ take a simple dislocation character-dependent form:

$$
\begin{gathered}
r_{0}(\theta)=r_{0}^{\text {edge }} \sin ^{2} \theta+r_{0}^{\text {screw }} \cos ^{2} \theta \\
E_{\text {core }}(\theta)=E_{\text {core }}^{\text {edge }} \sin ^{2} \theta+E_{\text {core }}^{\text {screw }} \cos ^{2} \theta
\end{gathered}
$$

where $\theta$ is the dislocation character angle: $\theta=0$ for pure screw and $\theta=\pi / 2$ for pure edge. Assume a semi-circular shape of the critical configuration, which is the most relevant to the critical shear stress, the line average of $r_{0}$ is given by

$$
\begin{aligned}
r_{0}^{\text {ave }} & =\frac{2}{\pi L} \int_{0}^{\frac{\pi L}{2}} r_{0}(\theta) \mathrm{d} l=\frac{4}{\pi L} \int_{0}^{\frac{\pi L}{4}} r_{0}(\theta) \mathrm{d} l=\frac{4}{\pi L} \int_{0}^{\frac{\pi}{2}} r_{0}(\theta) \cdot \frac{L}{2} \mathrm{~d} \theta \\
& =\frac{2}{\pi} \int_{0}^{\frac{\pi}{2}}\left(r_{0}^{\text {edge }} \sin ^{2} \theta+r_{0}^{\text {screw }} \cos ^{2} \theta\right) \mathrm{d} \theta=\frac{1}{2}\left(r_{0}^{\text {edge }}+r_{0}^{\text {screw }}\right)
\end{aligned}
$$

Similarly,

$$
E_{\text {core }}^{\text {ave }}=\frac{1}{2}\left(E_{\text {core }}^{\text {edge }}+E_{\text {core }}^{\text {screw }}\right)
$$

We remark that the line averages over character/orientation are approximation, which may yield less accurate results than direct DD simulations in which the dislocation core is a function of the character angle, as dictated by elasticity theory.

\section{References}

Aubry, S., Fitzgerald, S.P., Arsenlis, A., 2014. Methods to compute dislocation line tension energy and force in anisotropic elasticity. Modelling Simul. Mater. Sci. Eng. 22, 015001. URL: http://stacks . iop.org/0965-0393/22/i=1/a=015001, doi:10.1088/0965-0393/22/1/015001.

Bacon, D.J., 1967. A method for describing a flexible dislocation. Phys. Stat. Sol. (b) 23, 527-538. URL: http://onlinelibrary.wiley.com/doi/10.1002/pssb.19670230212/abstract, doi:10.1002/pssb. 19670230212. 
Bacon, D.J., Kocks, U.F., Scattergood, R.O., 1973. The effect of dislocation self-interaction on the Orowan stress. Philos. Mag. 28, 1241-1263. URL: http://dx.doi.org/10.1080/14786437308227997, doi:10 . 1080/14786437308227997.

Bacon, D.J., Osetsky, Y.N., Rodney, D., 2009. Dislocation-obstacle interactions at the atomic level, in: Kubin, L., Hirth, J.P. (Eds.), Dislocations in Solids. Elsevier. volume 15, pp. 1-90. URL: http://www.sciencedirect.com/science/article/pii/S1572485909015010.

Balint, D.S., Deshpande, V.S., Needleman, A., Van der Giessen, E., 2008. Discrete dislocation plasticity analysis of the grain size dependence of the flow strength of polycrystals. Int. J. Plast. 24, 2149-2172. URL: http://www.sciencedirect.com/science/article/pii/S0749641907001167, doi:10.1016/j.ijplas.2007.08.005.

Benzerga, A.A., 2008. An analysis of exhaustion hardening in micron-scale plasticity. Int. J. Plast. 24, 1128-1157. URL: http://www.sciencedirect.com/science/article/pii/S0749641907001209, doi:10.1016/j.ijplas.2007.08.010.

Benzerga, A.A., 2009. Micro-pillar plasticity: 2.5D mesoscopic simulations. J. Mech. Phys. Solids 57, 1459-1469. URL: http://www.sciencedirect.com/science/article/pii/S0022509609000908, doi:10.1016/j.jmps.2009.06.003.

Berry, J., Provatas, N., Rottler, J., Sinclair, C.W., 2014. Phase field crystal modeling as a unified atomistic approach to defect dynamics. Phys. Rev. B 89, 214117. URL: http://link.aps.org/doi/10.1103/PhysRevB.89.214117, doi:10.1103/PhysRevB.89.214117.

Bitzek, E., Gumbsch, P., 2004. Atomistic study of drag, surface and inertial effects on edge dislocations in face-centered cubic metals. Mater. Sci. Eng.: A 387-389, 11-15. URL: 
http://www.sciencedirect.com/science/article/pii/S0921509304004927, doi:10.1016/j.msea.2004.01.092.

Brown, L.M., 1964. The self-stress of dislocations and the shape of extended nodes. Philos. Mag. 10, 441466. URL: http://dx.doi.org/10.1080/14786436408224223, doi:10.1080/14786436408224223.

Brown, L.M., 1967. A proof of Lothe's theorem. Philos. Mag. 15, 363-370. URL: http://dx.doi.org/10.1080/14786436708227708, doi:10.1080/14786436708227708.

Cai, W., Arsenlis, A., Weinberger, C.R., Bulatov, V.V., 2006. A non-singular continuum theory of dislocations. J. Mech. Phys. Solids 54, 561-587. URL: http://www. sciencedirect.com/science/article/pii/S002250960500195X, doi:10.1016/j.jmps.2005.09.005.

Cai, W., Bulatov, V.V., Chang, J., Li, J., Yip, S., 2001. Anisotropic elastic interactions of a periodic dislocation array. Phys. Rev. Lett. 86, 57275730. URL: http://link.aps.org/doi/10.1103/PhysRevLett.86.5727, doi:10.1103/PhysRevLett.86.5727.

Cai, W., Bulatov, V.V., Chang, J., Li, J., Yip, S., 2005. Dislocation core effects on mobility, in: Hirth, J., Nabarro, F. (Eds.), Dislocations in Solids. Elsevier. volume 12, pp. 1-80. URL: http://www.sciencedirect.com/science/article/pii/S1572485905800038.

Cantrell, J.H., 2004. Substructural organization, dislocation plasticity and harmonic generation in cyclically stressed wavy slip metals. Proc. R. Soc. Lond. A 460, 757-780. URL: http://rspa.royalsocietypublishing.org/content/460/2043/757, doi:10.1098/rspa.2003.1181.

Chassagne, M., Legros, M., Rodney, D., 2011. Atomic-scale simulation of screw dislocation/coherent twin boundary interaction 
in $\mathrm{Al}, \mathrm{Au}, \mathrm{Cu}$ and $\mathrm{Ni}$ Acta Mater. 59, 1456-1463. URL:

http://www.sciencedirect.com/science/article/pii/S1359645410007512, doi:10.1016/j. actamat.2010.11.007.

Chen, Y., 2009. Reformulation of microscopic balance equations for multiscale materials modeling. J. Chem. Phys. 130, 134706. URL: http://jcp.aip.org/resource/1/jcpsa6/v130/i13/p134706_s1, doi:10.1063/1.3103887.

Cottrell, A.H., 2002. Commentary. A brief view of work hardening, in: Duesbery, M.S., Nabarro, F.R.N. (Eds.), Dislocations in Solids. Elsevier. volume 11, pp. vii-xvii. URL: http://www.sciencedirect.com/science/article/pii/S157248590280002X.

Crone, J.C., Munday, L.B., Knap, J., $2015 . \quad$ Capturing the effects of free surfaces on void strengthening with dislocation dynamics. Acta Mater. 101, 40-47. URL: http://www.sciencedirect.com/science/article/pii/S1359645415006540, doi:10.1016/j . actamat. 2015.08.067.

Deshpande, V.S., Needleman, A., Van der Giessen, E., 2005. Plasticity size effects in tension and compression of single crystals. J. Mech. Phys. Solids 53, 2661-2691. URL: http://www.sciencedirect.com/science/article/pii/S0022509605001274, doi:10.1016/j.jmps.2005.07.005.

deWit, G., Koehler, J.S., 1959. Interaction of dislocations with an applied stress in anisotropic crystals. Phys. Rev. 116, 11131120. URL: http://link.aps.org/doi/10.1103/PhysRev.116.1113, doi:10.1103/PhysRev.116.1113.

Fitzgerald, S.P., 2010. Frank-Read sources and the yield of anisotropic cubic crystals. Philos. Mag. Lett. 90, 209218. URL: http://dx.doi.org/10.1080/09500830903571392, doi:10. 1080/09500830903571392. 
Fitzgerald, S.P., Aubry, S., Dudarev, S.L., Cai, $\quad$ W., 2012. Dislocation dynamics simulation of Frank-Read sources in anisotropic $\alpha$-Fe. Modelling Simul. Mater. Sci. Eng. 20, 045022. URL: http://iopscience.iop.org/0965-0393/20/4/045022, doi:10.1088/0965-0393/20/4/045022.

Foreman, A.J.E., 1967. The bowing of a dislocation segment. Philos. Mag. 15, 1011-1021. URL: http://dx.doi.org/10.1080/14786436708221645, doi:10.1080/14786436708221645.

Frank, F.C., Read, W.T., $1950 . \quad$ Multiplication processes for slow moving dislocations. Phys. Rev. 79, 722-723. URL: $\quad$ http://link.aps.org/doi/10.1103/PhysRev.79.722, doi:10.1103/PhysRev.79.722.

Friedel, J., 1964. Dislocations. Pergamon Press.

Ghoniem, N.M., Tong, S.H., Sun, L.Z., 2000. Parametric dislocation dynamics: A thermodynamics-based approach to investigations of mesoscopic plastic deformation. Phys. Rev. B 61, 913927. URL: http://link.aps.org/doi/10.1103/PhysRevB.61.913, doi:10.1103/PhysRevB.61.913.

Gurrutxaga-Lerma, B., Balint, D.S., Dini, D., Sutton, A.P., 2015. The mechanisms governing the activation of dislocation sources in aluminum at different strain rates. J. Mech. Phys. Solids 84, 273-292. URL: http://www.sciencedirect.com/science/article/pii/S0022509615300715, doi:10.1016/j.jmps.2015.08.008.

Han, C.S., Wagoner, R.H., Barlat, F., 2004. On precipitate induced hardening in crystal plasticity: theory. Int. J. Plast. 20, 477-494. URL: http://www.sciencedirect.com/science/article/pii/S0749641903000986, doi:10.1016/S0749-6419(03)00098-6. 
Hartley, C.S., Mishin, Y., 2005. Characterization and visualization of the lattice misfit associated with dislocation cores. Acta Mater. 53, 1313-1321. URL: http://www.sciencedirect.com/science/article/pii/S1359645404007062, doi:10.1016/j.actamat.2004.11.027.

Hatano, T., 2006. Dynamics of a dislocation bypassing an impenetrable precipitate: The Hirsch mechanism revisited. Phys. Rev. B 74, 020102. URL: http://link.aps.org/doi/10.1103/PhysRevB.74.020102, doi:10.1103/PhysRevB.74.020102.

Hatano, T., Kaneko, T., Abe, Y., Matsui, H., 2008. Void-induced cross slip of screw dislocations in fcc copper. Phys. Rev. B 77, 064108. URL: http://link.aps.org/doi/10.1103/PhysRevB.77.064108, doi:10.1103/PhysRevB.77.064108.

Hatano, T., Matsui, H., 2005. Molecular dynamics investigation of dislocation pinning by a nanovoid in copper. Phys. Rev. B 72, 094105. URL: http://link.aps.org/doi/10.1103/PhysRevB.72.094105, doi:10.1103/PhysRevB.72.094105.

Hirth, J.P., Lothe, J., 1992. Theory of Dislocations. Krieger Pub Co.

Huang, M.S., Zhu, Y.X., Li, Z.H., 2014. Dislocation dissociation strongly influences on Frank-Read source nucleation and microplasticy of materials with low stacking fault energy. Chin. Phys. Lett. 31, 046102. URL: http://iopscience.iop.org/0256-307X/31/4/046102, doi:10.1088/0256-307X/31/4/046102.

Hull, D., Bacon, D.J., 2011. Introduction to Dislocations. 5th ed., ButterworthHeinemann.

de Koning, M., Cai, W., Bulatov, V.V., 2003. Anomalous dislocation multiplication in FCC metals. Phys. Rev. Lett. 91, 025503. URL: http://link.aps.org/doi/10.1103/PhysRevLett.91.025503, doi:10.1103/PhysRevLett.91.025503. 
Kuhlmann-Wilsdorf, D., 2002. The LES theory of solid plasticity, in: Nabarro, F.R.N., Duesbery, M. (Eds.), Dislocations in Solids. Elsevier. volume 11, pp. 211-342. URL: http://www.sciencedirect.com/science/article/pii/S1572485902800109.

Lee, D.W., Kim, H., Strachan, A., Koslowski, M., 2011. Effect of core energy on mobility in a continuum dislocation model. Phys. Rev. B 83, 104101. URL: http://link.aps.org/doi/10.1103/PhysRevB.83.104101, doi:10.1103/PhysRevB.83.104101.

Lehtinen, A., Granberg, F., Laurson, L., Nordlund, K., Alava, M.J., 2016. Multiscale modeling of dislocation-precipitate interactions in Fe: From molecular dynamics to discrete dislocations. Phys. Rev. E 93, 013309. URL: http://link.aps.org/doi/10.1103/PhysRevE.93.013309, doi:10.1103/PhysRevE.93.013309.

LeSar, R., 2004. Ambiguities in the calculation of dislocation self energies. Phys. Stat. Sol. (b) 241, 2875-2880. URL: http://onlinelibrary.wiley.com/doi/10.1002/pssb.200302054/abstract, doi:10.1002/pssb. 200302054.

Li, J., Wang, C.Z., Chang, J.P., Cai, W., Bulatov, V.V., Ho, K.M., Yip, S., 2004. Core energy and Peierls stress of a screw dislocation in bcc molybdenum: A periodic-cell tight-binding study. Phys. Rev. B 70, 104113. URL: http://link.aps.org/doi/10.1103/PhysRevB.70.104113, doi:10.1103/PhysRevB.70.104113.

Li, X.Y., Yang, W., 2006. Atomistic simulations for the evolution of a U-shaped dislocation in fcc Al. Phys. Rev. B 74, 144108. URL: http://link.aps.org/doi/10.1103/PhysRevB.74.144108, doi:10.1103/PhysRevB.74.144108.

Lothe, J., Hirth, J.P., $2005 . \quad$ Dislocation core parameters. $\quad$ Phys. Stat. Sol. (b) 242, 836-841. URL: 
http://onlinelibrary.wiley.com/doi/10.1002/pssb.200402114/abstract, doi:10.1002/pssb. 200402114.

Lubarda, V.A., Markenscoff, X., 2007. Configurational force on a lattice dislocation and the Peierls stress. Arch. Appl. Mech. 77, 147-154. URL: http://link. springer.com/article/10.1007/s00419-006-0068-y, doi:10.1007/s00419-006-0068-y.

Martin, J.W., 1998. Precipitation Hardening. 2nd ed., Butterworth-Heinemann.

Martínez, E., Marian, J., Arsenlis, A., Victoria, M., Perlado, J.M., 2008. Atomistically informed dislocation dynamics in fcc crystals. J. Mech. Phys. Solids 56, 869-895. URL: http://www.sciencedirect.com/science/article/pii/S0022509607001408, doi:10.1016/j.jmps.2007.06.014.

Mishin, Y., Farkas, D., Mehl, M.J., Papaconstantopoulos, D.A., 1999. Interatomic potentials for monoatomic metals from experimental data and $a b$ initio calculations. Phys. Rev. B 59, 33933407. URL: http://link.aps.org/doi/10.1103/PhysRevB.59.3393, doi:10.1103/PhysRevB.59.3393.

Mishin, Y., Mehl, M.J., Papaconstantopoulos, D.A., Voter, A.F., Kress, J.D., 2001. Structural stability and lattice defects in copper: Ab initio, tight-binding, and embedded-atom calculations. Phys. Rev. B 63, 224106. URL: http://link.aps.org/doi/10.1103/PhysRevB.63.224106, doi:10.1103/PhysRevB.63.224106.

Mott, N., Nabarro, F., 1948. Report of a Conference on Strength of Solids. volume 1. Physical Society.

Osetsky, Y.N., Bacon, D.J., 2003. An atomic-level model for studying the dynamics of edge dislocations in metals. Modelling Simul. Mater. Sci. Eng. 11, 427-446. URL: http://stacks.iop.org/0965-0393/11/i=4/a=302, doi:10.1088/0965-0393/11/4/302. 
Parrinello, M., Rahman, A., 1981. Polymorphic transitions in single crystals: A new molecular dynamics method. J. Appl. Phys. 52, 7182-7190. URL: http://scitation.aip.org/content/aip/journal/jap/52/12/10.1063/1.328693, doi:10.1063/1.328693.

Ramesh, K., 2009. Nanomaterials: Mechanics and Mechanisms. Springer, New York.

Ranganathan, S.I., Ostoja-Starzewski, M., 2008. Universal elastic anisotropy index. Phys. Rev. Lett. 101, 055504. URL: http://link.aps.org/doi/10.1103/PhysRevLett.101.055504, doi:10.1103/PhysRevLett.101.055504.

Scattergood, R.O., Bacon, D.J., $1982 . \quad$ The strengthening effect of voids. Acta Metall. 30, 1665-1677. URL: http://www.sciencedirect.com/science/article/pii/0001616082901882, doi:10.1016/0001-6160(82) 90188-2.

Schroeder, W., Martin, K., Lorensen, B., 2006. Visualization Toolkit: An Object-Oriented Approach to 3D Graphics. 4th ed., Kitware.

Shimokawa, T., Kitada, S., 2014. Dislocation multiplication from the Frank-Read source in atomic models. Mater. Trans. 55, 58-63. doi:10.2320/matertrans. MA201319.

Shishvan, S.S., Mohammadi, S., Rahimian, M., 2008. A dislocationdynamics-based derivation of the Frank-Read source characteristics for discrete dislocation plasticity. Modelling Simul. Mater. Sci. Eng. 16, 075002. URL: http://iopscience.iop.org/0965-0393/16/7/075002, doi:10.1088/0965-0393/16/7/075002.

Srinivasan, S.G., Liao, X.Z., Baskes, M.I., McCabe, R.J., Zhao, Y.H., Zhu, Y.T., 2005. Compact and dissociated dislocations in aluminum: Implications for deformation. Phys. Rev. Lett. 94, 125502. 
URL: http://link.aps.org/doi/10.1103/PhysRevLett.94.125502, doi:10.1103/PhysRevLett.94.125502.

Stukowski, A., 2010. Visualization and analysis of atomistic simulation data with OVITO - the Open Visualization Tool. Modelling Simul. Mater. Sci. Eng. 18, 015012. URL: http://iopscience.iop.org/0965-0393/18/1/015012, doi:10.1088/0965-0393/18/1/015012.

Stukowski, A., 2012. Structure identification methods for atomistic simulations of crystalline materials. Modelling Simul. Mater. Sci. Eng. 20, 045021. URL: http://iopscience.iop.org/0965-0393/20/4/045021, doi:10 . 1088/0965-0393/20/4/045021.

Stukowski, A., Bulatov, V.V., Arsenlis, A., 2012. Automated identification and indexing of dislocations in crystal interfaces. Modelling Simul. Mater. Sci. Eng. 20, 085007. URL: http://iopscience.iop.org/0965-0393/20/8/085007/cites, doi:10.1088/0965-0393/20/8/085007.

Szajewski, B.A., Chakravarthy, S.S., Curtin, W.A., 2013. Operation of a 3D Frank-Read source in a stress gradient and implications for size-dependent plasticity. Acta Mater. 61, 1469-1477. URL: http://www.sciencedirect.com/science/article/pii/S1359645412008270, doi:10.1016/j. actamat. 2012.11.023.

Szajewski, B.A., Curtin, W.A., 2015. Analysis of spurious image forces in atomistic simulations of dislocations. Modelling Simul. Mater. Sci. Eng. 23, 025008. URL: http://iopscience.iop.org/0965-0393/23/2/025008, doi:10 . 1088/0965-0393/23/2/025008.

Szajewski, B.A., Pavia, F., Curtin, W.A., 2015. Robust atomistic calculation of dislocation line tension. Modelling Simul. Mater. Sci. Eng. 23, 085008. URL: http://stacks.iop.org/0965-0393/23/i=8/a=085008, doi:10 . 1088/0965-0393/23/8/085008. 
Towns, J., Cockerill, T., Dahan, M., Foster, I., Gaither, K., Grimshaw, A., Hazlewood, V., Lathrop, S., Lifka, D., Peterson, G., Roskies, R., Scott, J., Wilkins-Diehr, N., 2014. XSEDE: Accelerating scientific discovery. Comput. Sci. Eng. 16, 62-74. doi:10.1109/MCSE. 2014.80.

Weertman, J., Weertman, J., 1992. Elementary Dislocation Theory. Oxford University Press, New York.

Xiang, Y., Cheng, L.T., Srolovitz, D.J., E, W., 2003. A level set method for dislocation dynamics. Acta Mater. 51, 5499-5518. URL: http://www.sciencedirect.com/science/article/pii/S1359645403004154, doi:10.1016/S1359-6454(03)00415-4.

Xiong, L., Deng, Q., Tucker, G., McDowell, D.L., Chen, Y., 2012a. A concurrent scheme for passing dislocations from atomistic to continuum domains. Acta Mater. 60, 899-913. URL: http://www.sciencedirect.com/science/article/pii/S1359645411007786, doi:10.1016/j. actamat.2011.11.002.

Xiong, L., McDowell, D.L., Chen, Y., 2012b. Nucleation and growth of dislocation loops in $\mathrm{Cu}, \mathrm{Al}$ and $\mathrm{Si}$ by a concurrent atomistic-continuum method. Scripta Mater. 67, 633-636. URL: http://www.sciencedirect.com/science/article/pii/S1359646212004691, doi:10.1016/j.scriptamat.2012.07.026.

Xiong, L., Rigelesaiyin, J., Chen, X., Xu, S., McDowell, D.L., Chen, Y., 2016. Coarse-grained elastodynamics of fast moving dislocations. Acta Mater. 104, 143-155. URL: http://www.sciencedirect.com/science/article/pii/S1359645415300884, doi:10.1016/j . actamat.2015.11.037.

Xiong, L., Tucker, G., McDowell, D.L., Chen, Y., 2011. Coarse-grained atomistic simulation of dislocations. J. Mech. Phys. Solids 59, 160-177. URL: http://www.sciencedirect.com/science/article/pii/S0022509610002395, doi:10.1016/j.jmps.2010.11.005. 
Xiong, L., Xu, S., McDowell, D.L., Chen, Y., $2015 . \quad$ Concurrent atomistic-continuum simulations of dislocation-void interactions in fcc crystals. Int. J. Plast. 65, 33-42. URL: http://www.sciencedirect.com/science/article/pii/S0749641914001508, doi:10.1016/j.ijplas.2014.08.002.

Xu, S., Che, R., Xiong, L., Chen, Y., McDowell, D.L., 2015. A quasistatic implementation of the concurrent atomistic-continuum method for FCC crystals. Int. J. Plast. 72, 91-126. URL: http://www.sciencedirect.com/science/article/pii/S0749641915000777, doi:10.1016/j.ijplas.2015.05.007.

Xu, S., Xiong, L., Chen, Y., McDowell, D.L., 2016a. Edge dislocations bowing out from a row of collinear obstacles in Al. Scripta Mater. 123, 135-139. URL: http://www.sciencedirect.com/science/article/pii/S135964621630272X, doi:10.1016/j.scriptamat.2016.06.018.

Xu, S., Xiong, L., Chen, Y., McDowell, D.L., 2016b. Sequential slip transfer of mixed-character dislocations across $\Sigma 3$ coherent twin boundary in FCC metals: a concurrent atomistic-continuum study. npj Comput. Mater.

2, 15016. URL: http://www.nature.com/articles/npjcompumats201516, doi:10.1038/npj compumats . 2015.16.

Xu, S., Xiong, L., Deng, Q., McDowell, D.L., 2016c. Mesh refinement schemes for the concurrent atomistic-continuum method. Int. J. Solids Struct. 90, 144-152. URL: http://www.sciencedirect.com/science/article/pii/S0020768316300154, doi:10.1016/j.ijsolstr.2016.03.030.

Zhang, R.F., Wang, J., Beyerlein, I.J., Germann, T.C., 2011. Dislocation nucleation mechanisms from fcc/bcc incoherent interfaces. Scripta Mater. 65, 1022-1025. URL: http://www.sciencedirect.com/science/article/pii/S1359646211005343, doi:10.1016/j.scriptamat. 2011.09.008. 
Zhang, R.F., Wang, J., Beyerlein, I.J., Misra, A., Germann, T.C., 2012. Atomic-scale study of nucleation of dislocations from fcc-bcc interfaces. Acta Mater. 60, 2855-2865. URL: http://www.sciencedirect.com/science/article/pii/S1359645412000882, doi:10.1016/j . actamat.2012.01.050. 